\title{
A COMPARISON OF CONSTRAINT QUALIFICATIONS IN INFINITE-DIMENSIONAL CONVEX PROGRAMMING REVISITED
}

\author{
C. ZĂLINESCU ${ }^{1}$
}

(Received 22 May 1997, revised 19 September 1997)

\section{Introduction}

In 1990 Gowda and Teboulle published the paper [16], making a comparison of several conditions ensuring the Fenchel-Rockafellar duality formula

$$
\inf \{f(x)+g(A x) \mid x \in X\}=\max \left\{-f^{*}\left(A^{*} y^{*}\right)-g^{*}\left(-y^{*}\right) \mid y^{*} \in Y^{*}\right\} .
$$

Probably the first comparison of different constraint qualification conditions was made by Hiriart-Urruty [17] in connection with $\varepsilon$-subdifferential calculus. Among them appears, as the basic sufficient condition, the formula for the conjugate of the corresponding function; such functions are: $f_{1}+f_{2}, g \circ A, \max \left\{f_{1}, \ldots, f_{n}\right\}$, etc. In fact strong duality formulae (like the one above) and good formulae for conjugates are equivalent and they can be used to obtain formulae for $\varepsilon$-subdifferentials, using a technique developed in [17] and extensively used in [46].

Meantime other papers treated similar problems, using other conditions. The aim of this note is to compare them with former conditions.

This paper is dedicated to Professors B. Craven and B. Mond on the occasion of their retirement.

\section{Interiority notions}

In establishing sufficient conditions for duality one uses several notions of interiority.

Let $X$ be a real linear space and $C \subset X$ a nonempty set. We recall that the algebraic interior (or core) of $C$ is

$$
C^{i}=\{x \in X \mid \forall y \in X, \exists \delta>0, \forall \lambda \in[-\delta, \delta]: x+\lambda y \in C\},
$$

\footnotetext{
${ }^{1}$ University “Al. I. Cuza” laşi, Faculty of Mathematics, Bd. Copou, Nr. 11, 6600 Iaşi, Romania (C) Australian Mathematical Society 1999, Serial-fee code 0334-2700/99
} 
that is, $x \in C^{i}$ if and only if $C-x$ is absorbing, while the algebraic relative interior (or intrinsic core) of $C$ is

$$
{ }^{i} C=\{x \in X \mid \forall y \in \text { aff } C, \exists \delta>0, \forall \lambda \in[-\delta, \delta]:(1-\lambda) x+\lambda y \in C\} .
$$

In the sequel span $C$, aff $C$, cone $C$ and conv $C$ represent the linear, affine, conical and convex hull of $C$, respectively; in particular cone $C=[0, \infty) \cdot C$.

When $X$ is a topological vector space (t.v.s. for short) we introduce the notation

$$
{ }^{i c} C= \begin{cases}{ }^{i} C & \text { if aff } C \text { is a closed manifold } \\ \emptyset & \text { otherwise }\end{cases}
$$

while for $X$ a locally convex space (l.c.s. for short) we consider

$$
{ }^{i b} C= \begin{cases}{ }^{i} C & \text { if } X_{0} \text { is a barreled linear subspace, } \\ \emptyset & \text { otherwise }\end{cases}
$$

where $X_{0}=\operatorname{span}(C-c)$ for some (every) $c \in C ; X_{0}$ is the linear subspace parallel to aff $C$. Recall that the l.c.s. $X$ is barreled if every closed, convex and absorbing subset is a neighborhood of the origin of $X$. The first notation is introduced in [49, p. A90].

If $X$ is a t.v.s. and $C \subset X$, int $C$, rint $C$ and $\bar{C}$ denote the interior, the interior with respect to aff $C$ and the closure of $C$, respectively. Related to rint $C$ and ${ }^{i c} C$ is ri $C$, the relative interior of $C$, introduced in [31, Definition 3.3.4]. More exactly

$$
\text { ri } C= \begin{cases}\operatorname{rint} C & \text { if aff } C \text { is a closed manifold, } \\ \emptyset & \text { otherwise }\end{cases}
$$

Of course, rint $C \subset{ }^{i} C$, and so rint $C=$ ri $C \subset{ }^{i c} C$ if aff $C$ is closed and rint $C \subset$ ${ }^{i b} C$ if the parallel space to aff $C$ is barreled. Let $C$ be a nonempty closed convex set; by a standard argument, if $X$ is a Fréchet space (that is, a complete metrizable l.c.s.) and aff $C$ is closed then rint $C=\operatorname{ri} C={ }^{i c} C$, while if $X$ is a l.c.s. and the parallel space to aff $C$ is barreled then rint $C={ }^{i b} C$. We shall see in the sequel other situations when these equalities hold.

In the sequel, in this section, $C \subset X$ is a convex set. It is easy to prove that

$$
\forall x \in C: \operatorname{span}(C-x)=\operatorname{cone}(C-C),
$$

whence aff $C=x+\operatorname{cone}(C-C)$ for every $x \in C$; therefore cone $(C-C)$ is the linear space parallel to aff $C$. Moreover

$$
x \in C^{i} \Leftrightarrow \forall y \in X, \exists \lambda>0:(1+\lambda) x-\lambda y \in C \Leftrightarrow \operatorname{cone}(C-x)=X
$$


and

$$
\begin{aligned}
x \in{ }^{i} C & \Leftrightarrow \forall y \in C, \exists \lambda>0:(1+\lambda) x-\lambda y \in C \\
& \Leftrightarrow \operatorname{cone}(C-x)=\operatorname{cone}(C-C) \\
& \Leftrightarrow \operatorname{cone}(C-x) \text { is a linear space } \\
& \Leftrightarrow \bigcup_{\lambda>0} \lambda(C-x) \text { is a linear space } \\
& \Leftrightarrow \bigcup_{n \in \mathbb{N}^{*}} n(C-x) \text { is a linear space. }
\end{aligned}
$$

Taking into account the characterizations of the elements of ${ }^{i} C$ given above, we see that

$$
\begin{aligned}
x \in{ }^{i b} C & \Leftrightarrow \operatorname{cone}(C-x) \text { is a barraled linear subspace of } X \\
& \Leftrightarrow \bigcup_{n \in \mathbb{N}^{*}} n(C-x) \text { is a barreled linear subspace of } X .
\end{aligned}
$$

The condition $0 \in{ }^{i b} C$ is used intensively by Simons [39] and the author [50, (H0)]. We have also that

$$
\begin{aligned}
x \in{ }^{i c} C & \Leftrightarrow \operatorname{cone}(C-x) \text { is a closed linear subspace of } X \\
& \Leftrightarrow \bigcup_{\lambda>0} \lambda(C-x) \text { is a closed linear subspace of } X .
\end{aligned}
$$

If $X$ is a Fréchet space and aff $C$ is closed then ${ }^{i c} C={ }^{i b} C$, but it is possible to have ${ }^{i b} C \neq \emptyset$ and ${ }^{i c} C=\emptyset$ (if aff $C$ is not closed).

We have that ${ }^{i c} C$ is nothing else but the strong quasi relative interior sqri $C$ introduced in [19]; sqri $C$ is used in $[2,16]$.

Prior to the introduction of sqri $C$, Borwein and Lewis [10] introduced the set qri $C$ of quasi relative interior points of $C ; x \in$ qri $C$ if $x \in C$ and $\overline{c o n e}(C-x)$ is a linear subspace of $X$. Taking into account that in a finite-dimensional separated t.v.s. the closure of a convex cone is a linear subspace if and only if itself is a linear subspace, it follows that in this case qri $C={ }^{i} C=$ sqri $C$ (see [10, Proposition 2.4] for the first equality).

Below we mention several properties of qri, some of them being slight refinements of those in [10]. First note that

$$
\begin{aligned}
x \in \text { qri } C & \Leftrightarrow x \in C \wedge \overline{\operatorname{cone}}(C-x)=\overline{\operatorname{cone}}(C-C) \\
& \Leftrightarrow x \in C \wedge x-C \subset \overline{\operatorname{cone}}(C-x),
\end{aligned}
$$

whence, taking into account that $\overline{\operatorname{cone}} A=\overline{\operatorname{con}} \bar{A}$ for $A \subset X$,

$$
\text { qri } C=C \cap \text { qri } \bar{C}, \quad{ }^{i} C \subset \text { qri } C \quad\left(\text { even } C \cap{ }^{i} \bar{C} \subset \text { qri } C \text { ), sqri } C \subset \text { qri } C .\right.
$$


It follows (see [10, Lemma 2.9] for $X$ a l.c.s.) that

$$
\forall x \in \text { qri } C, y \in C, \lambda \in[0,1):(1-\lambda) x+\lambda y \in \text { qri } C,
$$

and so, if qri $C \neq \emptyset$ then $\overline{\text { qri } C}=\bar{C}$ (see [10, Proposition 2.12] for $X$ a 1.c.s.).

Let $Y$ be another t.v.s. and $A \in \mathscr{L}(X, Y)$, where $\mathscr{L}(X, Y)$ denotes the class of continuous linear operators from $X$ into $Y$. Then $A$ (qri $C) \subset$ qri $A(C)$ (see [10, Proposition 2.21] for $X$ a l.c.s.). Indeed, if $x \in$ qri $C$ then, by (1), $x-C \subset \overline{c o n e}(C-x)$, whence

$$
\begin{aligned}
A x-A(C) & =A(x-C) \subset A(\overline{\text { cone }}(C-x)) \\
& \subset \overline{A(\operatorname{cone}(C-x))}=\overline{\text { cone }}(A(C)-A x),
\end{aligned}
$$

which shows that $A x \in$ qri $A(C)$. Moreover, if qri $C \neq \emptyset$, then

$$
A(\text { qri } C) \subset \text { qri } A(C) \subset \overline{A(\text { qri } C)} \text {. }
$$

Indeed, let $x_{0} \in$ qri $C$ and take $y \in$ qri $A(C)(\subset A(C))$. There exists $x \in C$ such that $y=A x$. From (2) it follows that $(1-\lambda) x_{0}+\lambda x \in$ qri $C$ for $\lambda \in(0,1)$. From the inclusion showed above we have that $(1-\lambda) A x_{0}+\lambda y \in$ qri $A(C)$ for $\lambda \in(0,1)$. Taking the limit as $\lambda \rightarrow 1$, we get $y \in \overline{A(\text { qri } C)}$.

From (3) we obtain that sqri $A(C) \subset \overline{A(\text { qri } C)}$, if qri $C \neq \emptyset$; this relation is shown in [16, Proposition 3.5] for $X$ a l.c.s. and $Y$ a Baire space. Also from (3) one obtains that $A$ (qri $C)={ }^{i}(A(C))$ if $A \in \mathscr{L}\left(X, \mathbf{R}^{n}\right)$ and qri $C \neq \emptyset$ (see [10, Proposition 2.10] for $X$ a l.c.s.); note that for a convex subset $B$ of $\mathbf{R}^{n}$ one has ${ }^{i} B={ }^{i} \bar{B}=$ qri $B$.

The relation $A$ (qri $C)={ }^{i}(A(C))$ mentioned above and the fact that qri $C$ happens to be nonempty, even when ${ }^{i} C$ is empty (in many situations in infinite dimensional spaces, as proven in [10]), show the usefulness of quasi relative interiors in partially finite convex programs.

The notion of quasi relative interior is related to that of united sets, as mentioned by Moussaoui and Volle [28]. In [21, Definition 1.7.5] and [24, Definition 7.6.5] one says that the convex sets $C_{1}, C_{2} \subset X$ are united if they cannot be properly separated, that is, if all closed hyper-planes which separate $C_{1}$ and $C_{2}$ contain both of them. In [28] it is shown that

$$
\text { qri } C=C \cap\{x \in X \mid\{x\} \text { and } C \text { are united }\} .
$$

Assume that $X$ is a separated l.c.s. If $\overline{c o n e}(C-x)(=\overline{\operatorname{cone}}(\bar{C}-x))$ is a linear subspace then $x \in \bar{C}$. Moreover, assuming that aff $C$ is closed and rint $C \neq \emptyset$, if $\overline{\operatorname{cone}}(C-x)$ is a linear subspace then $x \in$ rint $C$; when aff $C$ has finite codimension this statement follows from [28, Theorem III.2]. 
Indeed, the first statement follows by separating strictly $\{x\}$ and $\bar{C}$ if $x \notin \bar{C}$. For the second statement we may suppose that $x=0$. As aff $C$ is closed and $C \subset$ aff $C$, it follows that $0 \in \bar{C} \subset$ aff $C$; thus $X_{0}=$ aff $C$ is a linear subspace. If $0 \notin \operatorname{rint} C$, applying a separation theorem in $X_{0}$, we get $x_{0}^{*} \in X_{0}^{*}$ such that $\left\langle x, x_{0}^{*}\right\rangle>0$ for all $x \in$ rint $C$. Taking an extension $x^{*} \in X^{*}$ of $x_{0}^{*}$ we have that $0 \leq\left\langle x, x^{*}\right\rangle$ for every $x \in C$, and so $0 \leq\left\langle x, x^{*}\right\rangle$ for every $x \in=\overline{\text { cone }} C$. As $\overline{c o n e} C$ is a linear subspace we obtain that $0=\left\langle x, x^{*}\right\rangle$ for every $x \in \overline{\text { cone }} C$, contradicting the fact that $\left\langle x, x^{*}\right\rangle>0$ for all $x \in \operatorname{rint} C(\neq \emptyset)$. Therefore $x \in$ rint $C$.

\section{Subdifferentiability criteria}

Let $f: X \rightarrow \overline{\mathbf{R}}$; the domain of $f$ is $\operatorname{dom} f=\{x \in X \mid f(x)<\infty\}$, the epigraph of $f$ is epi $f=\{(x, t) \in X \times \mathbf{R} \mid f(x) \leq t\}$, while the sublevel set of $f$ at height $\lambda \in \mathbf{R}$ is the set $[f \leq \lambda]=\{x \in X \mid f(x) \leq \lambda\}$. The function $f: X \rightarrow \overline{\mathbf{R}}$ is proper if $\operatorname{dom} f \neq \emptyset$ and $f$ does not take the value $-\infty$.

In the sequel we denote by $\Lambda(X)$ the class of proper and convex functions $f$ : $X \rightarrow \overline{\mathbf{R}}$, and by $\Gamma(X)$ the class of lower semicontinuous (l.s.c. for short) functions $f \in \Lambda(X)$.

It is well known (see [27, Proposition 10.c], [24, Theorem 6.4.6], etc.) that the function $f \in \Lambda(X)$ is subdifferentiable at any $x \in \operatorname{dom} f$ where $f$ is continuous; it is also well known that $f$ is continuous at $x \in \operatorname{dom} f$ if and only if $f$ is bounded above on a neighborhood of $x$. Other sufficient conditions for subdifferentiability were obtained. We mention some of them, (almost) explicitly stated in the literature; another condition will follow from Theorem 3 in the next section (see Remark 3 ). We recall that $A \subset X$ is cs-closed if whenever $\left(\lambda_{n}\right)_{n \in \mathbb{N}^{*}} \subset[0, \infty), \sum_{n \geq 1} \lambda_{n}=1,\left(c_{n}\right)_{n \in \mathbb{N}^{*}} \subset C$ and the series $\sum_{n \geq 1} \lambda_{n} c_{n}$ is convergent with sum $x$, then $x \in C ; A$ is cs-complete if whenever $\left(\lambda_{n}\right)_{n \in \mathrm{N}^{*}} \subset[0, \infty), \sum_{n \geq 1} \lambda_{n}=1,\left(c_{n}\right)_{n \in \mathrm{N}^{*}} \subset C$ and $\left(\sum_{m=1}^{n} \lambda_{m} c_{m}\right)_{n \geq 1}$ is a Cauchy sequence, the series $\sum_{n \geq 1} \lambda_{n} c_{n}$ is convergent with sum $x \in C$. Of course, if $C$ is cs-complete then $C$ is cs-closed, and if $C$ is cs-closed then $C$ is convex. The function $f$ is cs-closed (cs-complete) if epi $f$ is cs-closed (cs-complete) in $X \times \mathbf{R}$.

If $f \in \Lambda(X)$ and $x_{0} \in \operatorname{dom} f$, taking $g \in \Lambda(X), g(x)=f\left(x_{0}+x\right)$, we have that $\partial f\left(x_{0}\right)=\partial g(0)$; so, without loss of generality, we may take $x_{0}=0$.

Proposition 1. Let $X$ be a l.c.s. and $f \in \Lambda(X)$. Each of the following statements ensures that $\partial f(0)$ is nonempty:

(i) there exists $\lambda_{0} \in(f(0), \infty)$ such that $0 \in \operatorname{rint}\left[f \leq \lambda_{0}\right]$ (or, equivalently, $0 \in \operatorname{dom} f$ and $\left.f\right|_{x_{0}}$ is continuous at 0 , where $X_{0}=\operatorname{span}(\operatorname{dom} f)$ );

(ii) $X$ is separated, $\operatorname{dim} X_{0}<\infty$ and $0 \in{ }^{i}(\operatorname{dom} f)$;

(iii) $f \in \Gamma(X)$ and $0 \in{ }^{i b}(\operatorname{dom} f)$; 
(iv) $f$ is cs-closed, $0 \in{ }^{i c}(\operatorname{dom} f)$ and $X$ is a Fréchet space.

Moreover, if $X$ is separated and $\operatorname{dim} X_{0}<\infty$ then ${ }^{i}(\operatorname{dom} f)=\operatorname{rint}(\operatorname{dom} f)$, if $f \in \Gamma(X)$ and $^{i b}(\operatorname{dom} f) \neq \emptyset$ then $^{i b}(\operatorname{dom} f)=\operatorname{rint}(\operatorname{dom} f)$, and if $f$ is cs-closed, ${ }^{i c}(\operatorname{dom} f) \neq \emptyset$ and $X$ is a Fréchet space then ${ }^{i c}(\operatorname{dom} f)=\operatorname{rint}(\operatorname{dom} f)$.

Proof. If (ii), (iii) or (iv) holds then $0 \in \operatorname{dom} f$ and $f_{0}=\left.f\right|_{x_{0}}$ is continuous at 0 ; in particular $0 \in \operatorname{rint}(\operatorname{dom} f$ ). Suppose that (i) holds. By what we said at the beginning of this section, $\partial f_{0}(0) \neq \emptyset$. Because $X$ is a l.c.s., every $\varphi \in \partial f_{0}(0)$ may be extended to $x^{*} \in X^{*}$ (by Hahn-Banach theorem). Of course such an $x^{*}$ is in $\partial f(0)$.

Taking $x_{0} \in{ }^{i}(\operatorname{dom} f)\left[\operatorname{or}^{i b}(\operatorname{dom} f)\right.$ or $\left.{ }^{i c}(\operatorname{dom} f)\right]$ and the function $\tilde{f}$ defined by $\tilde{f}(x)=f\left(x+x_{0}\right)$ for $x \in X$, in everyone of the three situations $\tilde{f}$ satisfies the conditions in (ii), (iii) and (iv), respectively. Therefore $0 \in \operatorname{int}(\operatorname{dom} \tilde{f})$, whence $x_{0} \in \operatorname{rint}(\operatorname{dom} f)$.

The next result is related to the preceding proposition.' Recall first that for $f \in \Lambda(X)$, the directional derivative of $f$ at $x_{0} \in \operatorname{dom} f$ is defined (and exists) by $f^{\prime}\left(x_{0} ; x\right)=\lim _{t \backslash 0}\left(f\left(x_{0}+t x\right)-f\left(x_{0}\right)\right) / t \in \overline{\mathbf{R}}$.

PROPOSITION 2. Suppose that the hypotheses of Proposition 1 hold. Then

$$
\sup \left\{\left\langle x, x^{*}\right\rangle \mid x^{*} \in \partial f(0)\right\} \begin{cases}=f^{\prime}(0 ; x) & \text { if } x \in X_{0}, \\ <f^{\prime}(0 ; x)=\infty & \text { if } x \in \bar{X}_{0} \backslash X_{0}, \\ =f^{\prime}(0 ; x)=\infty & \text { if } x \in X \backslash \bar{X}_{0}\end{cases}
$$

the supremum being attained for $x \in X_{0}$. Moreover, $X_{0}$ is closed if and only if

$$
\forall x \in X: f^{\prime}(0 ; x)=\sup \left\{\left\langle x, x^{*}\right\rangle \mid x^{*} \in \partial f(0)\right\} .
$$

PROOF. First of all note that, with the notation from the proof of Proposition 1, we have

$$
\partial f_{0}(0)=\left\{\left.x^{*}\right|_{x_{0}} \mid x^{*} \in \partial f(0)\right\} \text { and } f^{\prime}(0 ; x)= \begin{cases}f_{0}^{\prime}(0 ; x) & \text { if } x \in X_{0}, \\ \infty & \text { if } x \in X \backslash X_{0}\end{cases}
$$

Since $f_{0}$ is continuous at $0\left(\in X_{0}\right)$, we have that

$$
\forall x \in X_{0}: f_{0}^{\prime}(0 ; x)=\max \left\{\left\langle x, x^{*}\right\rangle \mid x^{*} \in \partial f_{0}(0)\right\},
$$

\footnotetext{
'The referee considered that "in Proposition 1, it could be interesting to know if the directional derivative of $f$ at 0 is equal to the support function of $\partial f(0)$. This is true assuming that $f \in \Gamma(X), X$ Banach and $0 \in{ }^{i c}(\operatorname{dom} f)$ (see [11])"; in the same conditions this result was obtained before in [43, Theorem 7].
} 
whence, for $\bar{x} \in X_{0}$,

$$
\begin{aligned}
& \sup \left\{\left\langle\bar{x}, x^{*}\right\rangle \mid x^{*} \in \partial f(0)\right\}=\sup \left\{\left(\bar{x}, x^{*}\left|x_{0}\right\rangle \mid x^{*} \in \partial f(0)\right\}\right. \\
& \quad=\sup \left\{\left\langle\bar{x}, x^{*}\right\rangle \mid x^{*} \in \partial f_{0}(0)\right\}=\max \left\{\left\langle\bar{x}, x^{*}\right\rangle \mid x^{*} \in \partial f_{0}(0)\right\} \\
& =f_{0}^{\prime}(0 ; \bar{x})=f^{\prime}(0 ; \bar{x}) .
\end{aligned}
$$

If $\bar{x} \notin \overline{X_{0}}=X_{0}^{\perp \perp}$, there exists $\bar{x}^{*} \in X^{*}$ such that $\left.\bar{x}^{*}\right|_{X_{0}}=0$ and $\left\langle\bar{x}, \bar{x}^{*}\right\rangle \neq 0$. Taking $\bar{x}_{0}^{*} \in \partial f(0)(\neq \emptyset$ by Proposition 1$)$, we obtain that

$$
\sup \left\{\left(\bar{x}, x^{*}\right\rangle \mid x^{*} \in \partial f(0)\right\} \geq \sup \left\{\left(\bar{x}, \bar{x}_{0}^{*}+t \bar{x}^{*}\right\rangle \mid t \in \mathbf{R}\right\}=\infty=f^{\prime}(0 ; \bar{x}) .
$$

Let now $\bar{x} \in \overline{X_{0}} \backslash X_{0}$. Since $f_{0}$ is continuous at 0 , by [44, Proposition 2], $f_{0}$ is Lipschitz at 0 , that is, there exists $V_{0}$ a closed, convex and symmetric neighborhood of $0 \in X_{0}$ such that $V_{0} \subset \operatorname{dom} f_{0}$ and $\left|f_{0}(x)-f_{0}(y)\right| \leq p_{V_{0}}(x-y)$ for all $x, y \in V_{0}$, where $p_{V_{0}}$ denotes the Minkowski functional associated to the set $V_{0}$. It follows that $f_{0}^{\prime}(0 ; x) \leq p_{V_{0}}(x)$ for all $x \in X_{0}$. Replacing eventually $V_{0}$ by a subset, we may suppose that $V_{0}=V \cap X_{0}$, where $V$ is a closed, convex and symmetric neighborhood of $0 \in X$. So we have that $f_{0}^{\prime}(0 ; x) \leq p_{V}(x)$ for all $x \in X_{0}$. It follows that

$$
\forall x \in X_{0}, \forall x^{*} \in \partial f(0):\left\langle x, x^{*}\right\rangle=\left\langle x,\left.x^{*}\right|_{x_{0}}\right\rangle \leq f_{0}^{\prime}(0 ; x) \leq p_{V}(x) .
$$

As $\bar{x} \in \overline{X_{0}}$, there exists a net $\left(x_{i}\right) \subset X_{0}$ converging to $\bar{x}$. From the relation above, taking into account the continuity of $x^{*} \in \partial f(0)$ and $p_{v}$, we obtain that $\left\langle\bar{x}, x^{*}\right\rangle \leq$ $p_{v}(x)$ for all $x^{*} \in \partial f(0)$, and so $\sup \left\{\left(\bar{x}, x^{*}\right\rangle \mid x^{*} \in \partial f(0)\right\} \leq p_{v}(\bar{x})<\infty$.

REMARK 1. Condition (i) is surely known by convex analysts, and explicitly stated in [51, Theorem 2.4.5] and [13, Lemma 3.1] (in this paper the neighborhood being taken for the Mackey topology). Condition (ii) is, practically, [35, Theorem 23.4]. In [7, Corollary 5.6] it is shown that $f$ is continuous if (iii) or (iv) holds and $X_{0}=X$; in [9, Theorem 1] one obtains again that $f$ is continuous under (iii) if $X_{0}=X$.

\section{The fundamental duality formula}

In [36] Rockafellar uses systematically the perturbation functions in order to derive optimality conditions and duality formulae. This approach is also used in $[5,12,13$, $15,32,46,47,49-51]$.

In the rest of the paper $X, Y, Z$ are separated locally convex spaces (s.l.c.s. for short), $\mathscr{V}_{X}$ denotes the class of convex neighborhoods of the origin in $X$ and $\mathscr{B}_{X}$ denotes the class of nonempty bounded subsets of $X$; recall that $B \subset X$ is bounded if for every $V \in \mathscr{V}_{X}$ there exists $\lambda>0$ such that $B \subset \lambda V$. 
Let $\Phi \in \Lambda(X \times Z)$. Using the definition of the conjugate or the Young-Fenchel inequality, we have

$$
\forall x \in X, z^{*} \in Z^{*}: \Phi(x, 0) \geq-\Phi^{*}\left(0, z^{*}\right),
$$

whence

$$
\inf \{\Phi(x, 0) \mid x \in X\} \geq \sup \left\{-\Phi^{*}\left(0, z^{*}\right) \mid z^{*} \in Z^{*}\right\} .
$$

In convex analysis the relation

$$
\inf \{\Phi(x, 0) \mid x \in X\}=\max \left\{-\Phi^{*}\left(0, z^{*}\right) \mid z^{*} \in Z^{*}\right\}
$$

is fundamental. So, it is very important to have sufficient conditions which ensure the validity of (4). Usually one considers the family of convex minimization problems

$$
\left(P_{z}\right) \quad \min \Phi(x, z), \quad x \in X,
$$

for $z \in Z$, and the corresponding marginal (or value) function

$$
h: Z \rightarrow \overline{\mathbf{R}}, \quad h(z)=\inf \{\Phi(x, z) \mid x \in X\} .
$$

It is known, and simple to prove, that $h$ is convex, $\operatorname{dom} h=\operatorname{Pr}_{Z}(\operatorname{dom} \Phi)$ and $h^{*}\left(z^{*}\right)=\Phi^{*}\left(0, z^{*}\right)$ for every $z^{*} \in Z^{*}$. In the sequel we shall suppose that $0 \in \operatorname{dom} h$, or equivalently, $0 \in \operatorname{Pr}_{Z}(\operatorname{dom} \Phi)$. In this situation $h(0) \in \mathbf{R}$ or $h(0)=-\infty$. Recall that if the convex function $f: X \rightarrow \overline{\mathbf{R}}$ takes the value $-\infty$ then $f$ is identically $-\infty$ on ${ }^{i}(\operatorname{dom} f)$ and $f^{*}$ is identically $+\infty$ on $X^{*}$. Therefore (4) holds if $h(0)=-\infty$. If $h(0) \in \mathbf{R},(4)$ is equivalent to $h(0)=-h^{*}\left(z^{*}\right)$ for some $z^{*} \in Z^{*}$, that is, $\partial h(0) \neq \emptyset$. Before stating the next theorem we introduce an hypothesis on sets (in product spaces) which is intermediate between cs-closedness and cs-completeness. Let $A \subset X \times Y$; we say that $A$ satisfies (Hx) (the letter $\mathrm{x}$ refers to the variable $x$ ) if

$$
\text { (Hx) }\left\{\begin{array}{l}
\forall\left(\lambda_{n}\right)_{n \geq 1} \subset[0, \infty),\left(\left(x_{n}, y_{n}\right)\right)_{n \geq 1} \subset A \text { with } \sum_{n \geq 1} \lambda_{n}=1, \sum_{n \geq 1} \lambda_{n} y_{n}=y, \\
\left(\sum_{k=1}^{n} \lambda_{k} x_{k}\right)_{n \geq 1} \text { a Cauchy sequence, } \exists x=\sum_{n \geq 1} \lambda_{n} x_{n} \text { and }(x, y) \in A .
\end{array}\right.
$$

Recall that $f \in \Lambda(X)$ is quasi-continuous (see [21, Definition 1.7.3], [24, Definition 7.6.3], the last one under the name of $d$-quasi-continuity) if aff (dom $f$ ) is closed with finite codimension and $\left.f\right|_{\text {aff(dom } f)}$ is continuous at every $x \in \operatorname{rint}(\operatorname{dom} f)$, supposed to be nonempty.

In the next theorem we state several sufficient conditions for the fundamental duality formula (4).

THEOREM 3. Let $\Phi \in \Lambda(X \times Z)$ be such that $0 \in \operatorname{Pr}_{Z}(\operatorname{dom} \Phi)$. Consider $Z_{0}=$ span $\left(\operatorname{Pr}_{Z}(\operatorname{dom} \Phi)\right)$. Each of the following conditions is sufficient for (4) : 
(i) there exists $\lambda_{0} \in \mathbf{R}$ such that $\left\{z \in Z \mid \exists x \in X, \Phi(x, z) \leq \lambda_{0}\right\} \in \mathscr{V}_{Z_{0}}$;

(ii) there exists $x_{0} \in X$ such that $\left(x_{0}, 0\right) \in \operatorname{dom} \Phi$ and $\Phi\left(x_{0}, \cdot\right)$ is continuous at 0 ;

(iii) $\operatorname{dim} Z_{0}<\infty$ and $0 \in{ }^{i}\left(\operatorname{Pr}_{Z}(\operatorname{dom} \Phi)\right)$;

(iv) $X$ is a Fréchet space, $\Phi \in \Gamma(X \times Z)$ and $0 \in{ }^{i b}\left(\operatorname{Pr}_{Z}(\operatorname{dom} \Phi)\right)$;

(v) $X, Z$ are metrizable, $0 \in{ }^{i b}\left(\operatorname{Pr}_{Z}(\operatorname{dom} \Phi)\right)$ and epi $\Phi$ satisfies $(\mathrm{Hx})$;

(vi) $X, Z$ are Fréchet spaces, $\Phi \in \Gamma(X \times Z)$ and $0 \in{ }^{i c}\left(\operatorname{Pr}_{Z}(\operatorname{dom} \Phi)\right)$;

(vii) there exists $x_{0} \in X$ such that $\Phi\left(x_{0}, \cdot\right)$ is quasi-continuous and $\{0\}$ and $\operatorname{Pr}_{Z}(\operatorname{dom} \Phi)$ are united.

PROOF. (i) says that the marginal function $h$ satisfies condition (i) of Proposition 1. Therefore (4) holds.

(ii) $\Rightarrow$ (i) Of course, if (ii) is satisfied then $Z_{0}=Z$ and (i) holds with $\lambda_{0}=$ $\Phi\left(x_{0}, 0\right)+1$.

If (iii) is satisfied then $h$ satisfies condition (ii) of Proposition 1 (so (i) holds).

If (iv) holds, taking $\Phi_{0}: X \times Z_{0} \rightarrow \overline{\mathbf{R}}, \Phi_{0}(x, z)=\Phi(x, z)$, the conditions of [46, Theorem 4.3] are satisfied. Therefore (i) is valid (even the stronger condition (5) below).

(v) is slightly stronger than the hypotheses of [50, Proposition 3]; therefore the conclusion holds.

(vi) $\Rightarrow$ (iv) and (vi) $\Rightarrow$ (v) are obvious.

(vii) $\Rightarrow$ (i) By [21, Proposition 1.7.4] or [24, Proposition 7.6.4], $h$ is quasicontinuous, and so $\operatorname{rint}(\operatorname{dom} h) \neq \emptyset$. Since $\{0\}$ and $\operatorname{dom} h$ are united, the discussion from the end of Section 2 shows that $0 \in \operatorname{rint}(\operatorname{dom} h)$, and so $\left.h\right|_{z_{0}}$ is continuous at 0 , that is, (i) holds.

REMARK 2. Condition (ii) may be found in almost all books and articles dealing with perturbation functions. See, for example, [21, Theorem 1.7.1], [24, Theorem 7.6.1], [15, Proposition 2.3], [36, Theorem 18(a)]. Condition (vii) is stated by Joly and Laurent [21, Theorem 1.7.7] (see also [24, Theorem 7.6.7]) for $\Phi$ 1.s.c., and by Moussaoui and Volle [28, Theorem IV.1] for arbitrary $\Phi$; note that in $[21,24]$ there are also other conditions implying (vii)]. Condition (iii) is explicitly stated in [36, Theorem 18(b)] for $Z_{0}=Z$, in [21, Theorem 1.7.9], [24, Theorem 7.6.9] for $\operatorname{dim} Z<\infty$ and in [51, Theorem 2.6.5 (iv)]. Condition (i) with $Z_{0}=Z$ is [36, Theorem 18 (a)], and, in the present form, may be found in [51, Theorem 2.6 .5 (i)] and [13, Theorem 3.2]. As mentioned in the proof, condition (iv) is established in [46, Theorem 4.3] for $Z_{0}=Z$ (and $R$ replaced by a s.l.c.s. ordered by a normal cone), while condition ( $v$ ) is given in [50, Proposition 3]. Condition (vi) seems to be first used, in the present form, in [49, Theorem 6]. It is obtained again by Jeyakumar [18, Proposition 3.1]; he uses sqri $\left(P_{Z}(\operatorname{dom} \Phi)\right)$ instead of ${ }^{i c}\left(\operatorname{Pr}_{Z}(\operatorname{dom} \Phi)\right)$, but these sets 
coincide. For $X$ and $Z$ Banach spaces and $Z_{0}=Z$ condition (vi) is established by Rockafellar [36, Theorem 18 (c)] for $X$ reflexive, and Robinson [32, Corollary 1]; it is also obtained in [4, Theorem 3.1], and [6, Theorem A2].

REMARK 3. Note that every convex function $f: Z \rightarrow \overline{\mathbf{R}}$ may be viewed as a marginal function; just take $X=\{0\}$ and $\Phi: X \times Z \rightarrow \overline{\mathbf{R}}, \Phi(x, z)=f(z)$. Therefore every condition from Theorem 3 gives a subdifferentiability criterion. The new ones (in comparison with Proposition 1) are given by: epi $f$ is cs-closed and $0 \in{ }^{i b}(\operatorname{dom} f)$, and $f$ is quasi-continuous and $\{0\}$ and $\operatorname{dom} f$ are united (in particular if $0 \in \operatorname{qri}(\operatorname{dom} f))^{2}$. The last situation, even not explicitly stated, can be found in $[21,24]$.

In applications it is important to have conditions on $\Phi$ which ensure that also the functions $\widetilde{\Phi}, \widetilde{\Phi}(x, z)=\Phi(x, z)-\left\langle x, x^{*}\right\rangle$ with $x^{*} \in X^{*}$, satisfy them. Such conditions are (ii)-(vii) from Theorem 3 .

Other conditions of this type are:

$$
\begin{aligned}
& \exists \lambda_{0} \in \mathbf{R}, x_{0} \in X, \forall U \in \mathscr{V}_{X}:\left\{z \in Z \mid \exists x \in x_{0}+U, \Phi(x, z) \leq \lambda_{0}\right\} \in \mathscr{V}_{z_{0}}, \\
& \exists \lambda_{0} \in \mathbf{R}, B \in \mathscr{B}_{X}, V_{0} \in \mathscr{V}_{Z_{0}}: V_{0} \subset\left\{z \in Z \mid \exists x \in B, \Phi(x, z) \leq \lambda_{0}\right\}, \\
& \forall U \in \mathscr{V}_{X}, \exists \lambda>0:\{z \in Z \mid \exists x \in \lambda U, \Phi(x, z) \leq \lambda\} \in \mathscr{V}_{Z_{0}},
\end{aligned}
$$

where, as in Theorem 3, $Z_{0}=\operatorname{span}\left(\operatorname{Pr}_{Z}(\operatorname{dom} \Phi)\right)$.

Condition (5) (for $Z_{0}=Z$ and $\mathbf{R}$ replaced by a s.l.c.s. ordered by a normal cone) is stated in [46, (4.2')], while condition (6) is stated in [51, Theorem 2.6.5 (i')]. Condition (7) is inspired by the conditions used in [13].

Proposition 4. Let $\Phi \in \Lambda(X \times Z)$. Then (5) $\Leftrightarrow(7) \Rightarrow(6)$, and (6) $\Rightarrow$ (7) if $X$ is a normed vector space.

Conditions (i)-(vi) being those of Theorem 3, we have: (ii) $\Rightarrow$ (6), (vi) $\Rightarrow$ (iv) $\Rightarrow$ (5), (iii) $\Rightarrow$ (6) and (vi) $\Rightarrow$ (v) $\Rightarrow$ (i).

Moreover, taking $D=\operatorname{Pr}_{Z}(\operatorname{dom} \Phi)$, one has: if $\operatorname{dim}(\operatorname{span} D)<\infty$ then ${ }^{i} D=$ rint $D$; if $\Phi$ is l.s.c., $X$ is a Fréchet space and ${ }^{i b} D \neq \emptyset$ then ${ }^{i b} D=\operatorname{rint} D$; if $\Phi$ is l.s.c., $X, Z$ are Fréchet spaces and ${ }^{i c} D \neq \emptyset$ then ${ }^{i c} D=$ rint $D$.

Proof. (5) $\Rightarrow$ (7) Consider $\lambda_{0} \in \mathbf{R}$ and $x_{0} \in X$ given by (5). Let $U \in \mathscr{V}_{X}$. There exists $\mu>0$ such that $x_{0} \in \mu U$. Let $V=\left\{z \in Z \mid \exists x \in x_{0}+U, \Phi(x, z) \leq\right.$ $\left.\lambda_{0}\right\} \in \mathscr{V}_{z_{0}}$. Taking $\lambda=\max \left\{\lambda_{0}, \mu+1\right\}$ and $z \in V$, there exists $x \in x_{0}+U$ such that $\Phi(x, z) \leq \lambda_{0}$. As $x \in x_{0}+U \subset \mu U+U=(\mu+1) U \subset \lambda U$, the conclusion follows.

\footnotetext{
${ }^{2}$ This is related to referee's remark: "in [28], Moussaoui and Volle proved that $\partial f(0)$ is non void assuming that $f$ is quasi-continuous with $0 \in$ qri $(\operatorname{dom} f)$ ".
} 
(7) $\Rightarrow(5)$ It is obvious that there exists $x_{0} \in X$ such that $\Phi\left(x_{0}, 0\right)<\infty$. Consider $\lambda_{0}=\max \left\{\Phi\left(x_{0}, 0\right), 0\right\}+1$ and let $U \in \mathscr{V}_{X}$. There exists $U_{0} \in \mathscr{V}_{X}$ symmetric such that $U_{0}+U_{0} \subset U$. There exists $\lambda_{1}>0$ such that $x_{0} \in \lambda_{1} U_{0}$. By hypothesis, there exists $\lambda \geq \lambda_{0}+\lambda_{1}$ such that $V_{0}=\left\{z \in Z \mid \exists x \in \lambda U_{0}, \Phi(x, z) \leq \lambda\right\} \in \mathscr{V}_{z_{0}}$. Let $V=\lambda^{-1} V_{0}$ and take $z \in V$. As $\lambda z \in V_{0}$, there exists $x^{\prime} \in \lambda U_{0}$ such that $\Phi\left(x^{\prime}, \lambda z\right) \leq \lambda$. It follows that

$$
\Phi\left(\left(1-\lambda^{-1}\right)\left(x_{0}, 0\right)+\lambda^{-1}\left(x^{\prime}, \lambda z\right)\right) \leq\left(1-\lambda^{-1}\right) \Phi\left(x_{0}, 0\right)+\lambda^{-1} \Phi\left(x^{\prime}, \lambda z\right) \leq \lambda_{0},
$$

whence $\Phi(x, z) \leq \lambda_{0}$, where $x=x_{0}+\lambda^{-1}\left(x^{\prime}-x_{0}\right) \in x_{0}+U_{0}+U_{0} \subset x_{0}+U$.

(6) $\Rightarrow$ (7) Let $U \in \mathscr{V}_{X}$; there exists $\mu>0$ such that $B \subset \mu U$. Taking $\lambda=$ $\max \left\{\lambda_{0}, \mu\right\}$ we have that

$$
\begin{aligned}
\left\{z \in Z \mid \exists x \in B, \Phi(x, z) \leq \lambda_{0}\right\} & \subset\left\{z \in Z \mid \exists x \in \mu U, \Phi(x, z) \leq \lambda_{0}\right\} \\
& \subset\{z \in Z \mid \exists x \in \lambda U, \Phi(x, z) \leq \lambda\} .
\end{aligned}
$$

The conclusion follows.

(7) $\Rightarrow(6)$ when $X$ is a normed vector space. Take $U=U_{X}=\{x \in X \mid\|x\| \leq 1\}$. There exists $\lambda_{0}>0$ such that $\left\{z \in Z \mid \exists x \in \lambda_{0} U, \Phi(x, z) \leq \lambda_{0}\right\} \in \mathscr{V}_{z_{0}}$. As $\lambda_{0} U$ is bounded, the conclusion follows.

It is obvious that (ii) implies (6) with $B=\left\{x_{0}\right\}$, while (vi) $\Rightarrow$ (iv) is evident. As observed in the proof of Theorem 3, (iv) $\Rightarrow(5)$. The implication (v) $\Rightarrow$ (i) is shown in the proof of [50, Proposition 3].

Suppose that $\operatorname{dim} Z_{0}<\infty$ and $0 \in{ }^{i}\left(\operatorname{Pr}_{Z}(\operatorname{dom} \Phi)\right)$. It follows that there exist $z_{1}, \ldots, z_{m} \in \operatorname{Pr}_{Z}(\operatorname{dom} \Phi)$ such that $V_{0}=\operatorname{conv}\left\{z_{1}, \ldots, z_{m}\right\} \in \mathscr{V}_{z_{0}}$. For every $i$, $1 \leq i \leq m$, there exists $x_{i} \in X$ such that $\left(x_{i}, z_{i}\right) \in \operatorname{dom} \Phi$. Let $\lambda_{0}=\max \left\{\Phi\left(x_{i}, z_{i}\right) \mid\right.$ $1 \leq i \leq m\}$ and $B=\operatorname{conv}\left\{x_{1}, \ldots, x_{m}\right\}$. It is obvious that $B$ is bounded and for $z \in V_{0}$ there exist $\lambda_{1}, \ldots, \lambda_{m} \geq 0$ with $\sum_{i=1}^{m} \lambda_{i}=1$ such that $z=\sum_{i=1}^{m} \lambda_{i} z_{i}$. Then $x=\sum_{i=1}^{m} \lambda_{i} x_{i} \in B$ and $\Phi(x, z) \leq \lambda_{0}$. Hence (iii) $\Rightarrow(6)$.

The fact that ${ }^{i} D=\operatorname{rint} D$ if $\operatorname{dim}($ span $D)<\infty$ is obvious. Let $\Phi$ be l.s.c., $X$ be a Fréchet space and consider $z_{0} \in{ }^{i b} D$. Taking $\Phi_{0}$ defined by $\Phi_{0}(x, z)=\Phi\left(x, z+z_{0}\right)$, we have that $0 \in{ }^{i b} \operatorname{Pr}_{Z}\left(\operatorname{dom} \Phi_{0}\right)$. Of course $\Phi_{0}$ verify (iv) of Theorem 3. As remarked above, condition (5) holds, which implies that $0 \in \operatorname{rint}\left(\operatorname{Pr}_{Z}\left(\operatorname{dom} \Phi_{0}\right)\right)$, that is, $z_{0} \in$ rint $D$. Similarly one obtains the other relation.

We state another duality formula which will be useful in the sequel.

THEOREM 5. Let $F \in \Lambda(X \times Y), \mathscr{A} \subset X \times Y$ a nonempty convex set, and let $D=\cup\{\mathscr{A}(x)-y \mid(x, y) \in \operatorname{dom} F\}$, where $\mathscr{A}(x)=\{y \in Y \mid \exists x \in X,(x, y) \in \mathscr{A}\}$. Assume that $0 \in D$ and let $Y_{0}=\operatorname{span} D$. If one of the following conditions holds:

(i) for every $U \in \mathscr{V}_{X}$ there exist $\lambda>0$ and $V_{0} \in \mathscr{V}_{Y_{0}}$ such that

$$
\{0\} \times V_{0} \subset \mathscr{A} \cap(\lambda U \times Y)-[F \leq \lambda] \text {; }
$$


(ii) there exist $\lambda_{0} \in \mathbf{R}, B \in \mathscr{B}_{X}$ and $V_{0} \in \mathscr{V}_{Y_{0}}$ such that

$$
\{0\} \times V_{0} \subset \mathscr{A} \cap(B \times Y)-\left[F \leq \lambda_{0}\right] \text {; }
$$

(iii) $\operatorname{dim} Y_{0}<\infty$ and $0 \in{ }^{i} D$;

(iv) there exists $\left(x_{0}, y_{0}\right) \in \mathscr{A} \cap \operatorname{dom} F$ such that $F\left(x_{0}, \cdot\right)$ is continuous at $y_{0}$;

(v) $X, Y$ are metrizable, [( $F$ is cs-complete and $\mathscr{A}$ is cs-closed) or ( $F$ is cs-closed and $\mathscr{A}$ is cs-complete) $]$ and $0 \in{ }^{i b} D$;

(vi) $X, Y$ are Fréchet spaces, $F$ is l.s.c., $\mathscr{A}$ is closed and $0 \in{ }^{i b} D$, then there exists $z^{*} \in Y^{*}$ such that

$$
\inf \{F(x, y) \mid(x, y) \in \mathscr{A}\}=\inf \left\{F(x, y)+\left(z, z^{*}\right\rangle \mid(x, y+z) \in \mathscr{A}\right\} .
$$

Proof. Let $Z=Y$ and

$$
\Phi:(X \times Y) \times Z \rightarrow \overline{\mathbf{R}}, \quad \Phi(x, y ; z)= \begin{cases}F(x, y) & \text { if } y+z \in \mathscr{A}(x), \\ \infty & \text { otherwise }\end{cases}
$$

It follows easily that $\Phi$ is convex and $\operatorname{Pr}_{z}(\operatorname{dom} \Phi)=D$. It is obvious that the conclusion of the theorem is equivalent to $\inf _{(x, y) \in X \times Y} \Phi(x, y ; 0)=\max _{z^{*} \in Z^{*}}-\Phi^{*}\left(0,0 ; z^{*}\right)$. So we have to show that if one of the conditions of the theorem is verified then a condition of Theorem 3 holds.

If (i) holds it is immediate that $\Phi$ verifies condition (i) of Theorem 3. The implication (ii) $\Rightarrow$ (i) is obvious. Similar to the proof in Proposition 4 we have that (iii) $\Rightarrow$ (ii). We have also that (iv) $\Rightarrow$ (ii); just take $B=\left\{x_{0}\right\}, \lambda_{0}=F\left(x_{0}, y_{0}\right)+1$ and $V_{0}=\left\{y \in Y \mid F\left(x_{0}, y_{0}+y\right) \leq \lambda_{0}\right\}$ (in this case $Y_{0}=Y$ ).

If (vi) holds, it is clear that $\Phi$ satisfies condition (iv) of Theorem 3. By Proposition 4 it follows that relation (7) holds. Therefore for $U \times Y \in \mathscr{V}_{X \times Y}$ there exist $\lambda>0$ and $V \in \mathscr{V}_{Y_{0}}$ such that

$$
\begin{aligned}
& V \subset\{z \in Y \mid \exists(x, y) \in \lambda U \times Y, \Phi(x, y ; z) \leq \lambda\} \\
& \quad=\{z \in Y \mid \exists x \in \lambda U,(x, y+z) \in \mathscr{A}, F(x, y) \leq \lambda\},
\end{aligned}
$$

which means that (i) holds.

If $(v)$ holds then the conclusion is given by [50, Proposition 5].

Note that every condition of the preceding theorem is verified by $\widetilde{F}, \widetilde{F}(x, y)=$ $F(x, y)-\left\langle x, x^{*}\right\rangle$, where $x^{*} \in X^{*}$, when it is verified by $F$. Conditions (i) and (ii) are inspired by the conditions used in [13].

Taking $\mathscr{A}=X \times\{0\}$, the conclusion of the preceding theorem is just the conclusion of Theorem 3. Conditions (i), ii), (iii) and (iv) become (7), (6), (iii) and (ii) of Theorem 3, respectively; to conditions ( $v$ ) and (vi) correspond slightly stronger forms of conditions ( $v$ ) and (vi) of Theorem 3 , respectively. 
REMARK 4. If $F(x, y)=f(x)+g(y)$ with $f \in \Lambda(X), g \in \Lambda(Y)$, for condition (i) of Theorem 5 it is sufficient (and necessary if $f, g$ have proper conjugates) to have

$$
\forall U \in \mathscr{V}_{X}, \exists \lambda>0, V \in \mathscr{Y}_{Y_{0}}: V \subset[g \leq \lambda]-\mathscr{A}(\lambda U \cap[f \leq \lambda]),
$$

while for condition (ii) of Theorem 5 it is sufficient (and necessary if $f, g$ have proper conjugates) to have

$$
\exists \lambda_{0} \in \mathbf{R}, B \in \mathscr{B}_{X}, \quad V_{0} \in \mathscr{V}_{Y_{0}}: V_{0} \subset\left[g \leq \lambda_{0}\right]-\mathscr{A}\left(B \cap\left[f \leq \lambda_{0}\right]\right) .
$$

Of course, these two conditions are equivalent if $X$ is a normed space.

\section{Generalized Fenchel-Rockafellar duality}

In control problems appear functions of the type: $x \mapsto F(x, A x)$, where $F$ : $X \times Y \rightarrow \overline{\mathbf{R}}$ is a convex function and $A \in \mathscr{L}(X, Y)$. It is important to have formulae for the conjugate and $\varepsilon$-subdifferential of $\varphi_{1}: X \rightarrow \overline{\mathbf{R}}, \varphi_{1}(x)=F(x, A x)$. Such functions are considered, for example, in [15, Section 3.4], [3, Section 5.2].

THEOREM 6. Let $F \in \Lambda(X \times Y), A \in \mathscr{L}(X, Y)$ and $\varphi_{1}$ defined above. Assume that $0 \in D=\{A x-y \mid(x, y) \in \operatorname{dom} F\}$ and take $Y_{0}=\operatorname{span} D$. If one of the following conditions holds:

(i) there exist $\lambda_{0} \in \mathbf{R}, V_{0} \in \mathscr{V}_{Y_{0}}(0)$ and $B \in \mathscr{B}_{X}$ such that

$$
\{0\} \times V_{0} \subset\{(x, A x) \mid x \in B\}-\left[F \leq \lambda_{0}\right]
$$

(ii) for every $U \in \mathscr{V}_{X}$ there exist $\lambda>0$ and $V \in \mathscr{V}_{Y_{0}}$ such that

$$
\{0\} \times V \subset\{(x, A x) \mid x \in \lambda U\}-[F \leq \lambda] ;
$$

(iii) $\operatorname{dim} Y_{0}<\infty$ and $0 \in{ }^{i} D$;

(iv) there exists $x_{0} \in X$ such that $\left(x_{0}, A x_{0}\right) \in \operatorname{dom} F$ and $F\left(x_{0}, \cdot\right)$ is continuous at $A x_{0}$;

(v) $X$ is a Fréchet space, $F \in \Gamma(X \times Y)$ and $0 \in{ }^{i b} D$;

(vi) $X$ and $Y$ are metrizable, [( $F$ is cs-closed and $\operatorname{gr} A$ is cs-complete) or $F$ is cs-complete $]$ and $0 \in{ }^{i b} D$;

(vii) $X$ and $Y$ are Fréchet spaces, $F \in \Gamma(X \times Y)$ and $0 \in{ }^{i c} D$, then

$$
\begin{aligned}
\inf \{F(x, A x) \mid x \in X\} & =\max \left\{-F^{*}\left(A^{*} y^{*},-y^{*}\right) \mid y^{*} \in Y^{*}\right\} \\
\forall x^{*} \in X^{*}: \varphi_{1}^{*}\left(x^{*}\right) & =\min \left\{F^{*}\left(x^{*}-A^{*} y^{*}, y^{*}\right) \mid y^{*} \in Y^{*}\right\}, \\
\forall x \in \operatorname{dom} \varphi_{1}, \varepsilon \geq 0: \partial_{\varepsilon} \varphi_{1}(x) & =\left\{A^{*} y^{*}+x^{*} \mid\left(x^{*}, y^{*}\right) \in \partial_{\varepsilon} F(x, A x)\right\} .
\end{aligned}
$$


Proof. Consider $\Phi: X \times Y \rightarrow \overline{\mathbf{R}}, \Phi(x, y)=F(x, A x-y)$. Then $\Phi$ satisfies one of the conditions (6), (7), (ii)-(vi) of Theorem 3 when $F$ satisfies one of the conditions (i)-(vii), respectively. Applying Theorem 3 for the application $(x, y) \mapsto$ $\Phi(x, y)-\left\langle x, x^{*}\right\rangle$, one obtains (9). For (10) one uses the characterization of $\partial_{\varepsilon} \varphi_{1}(x)$ : $x^{*} \in \partial_{\varepsilon} \varphi_{1}(x)$ if and only if $x \in \operatorname{dom} \varphi_{1}$ and $\varphi_{1}(x)+\varphi_{1}^{*}\left(x^{*}\right) \leq\left\langle x, x^{*}\right\rangle+\varepsilon$.

Note that the conclusion of the theorem [excepting $(v)$ ] follows also from Theorem 5 for $F$ replaced by $F-x^{*}$ and $\mathscr{A}$ replaced by gr $A$.

Note also that (iii) $\Rightarrow$ (i) $\Rightarrow$ (ii), (iv) $\Rightarrow$ (i), (vii) $\Rightarrow$ (v) $\Rightarrow$ (ii) and (vii) $\Rightarrow$ (vi).

REMARK 5. Condition (iv) is used by Ekeland and Temam in [15, Theorem 4.1] to obtain (8), by Tiba [42] [for $X, Y$ reflexive Banach spaces and $F$ l.s.c.] and Zălinescu [45] to obtain (10) for $\varepsilon=0$. Condition (vi) is used in [50, Corollary 2] to obtain (9). Condition (vii) (with $Y_{0}=Y$ ) may be found in [47, Theorem 4 (9)] and conditions (i), (iii), (iv), (vii) (in Banach spaces) are considered in [51, Theorem 2.7.1] for the same conclusions.

Proposition 7. Let $F \in \Lambda(X \times Y), A \in \mathscr{L}(X, Y)$ and $D=\{A x-y \mid(x, y) \in$ $\operatorname{dom} F\}$. If $\operatorname{dim}(\operatorname{span} D)<\infty$ then $^{i} D=\operatorname{rint} D$; if $X$ is a Fréchet space, $F$ is l.s.c. and ${ }^{i b} D \neq \emptyset$ then ${ }^{i b} D=\operatorname{rint} D$; if $X, Y$ are metrizable, $[(F$ is cs-closed and $\operatorname{gr} A$ is cs-complete) or $F$ is cs-complete] and ${ }^{i b} D \neq \emptyset$ then ${ }^{i b} D=$ rint $D$; if $X, Y$ are Fréchet spaces, $F$ is l.s.c. and ${ }^{i c} D \neq \emptyset$ then ${ }^{i c} D=$ rint $D$.

Moreover, suppose that $X, Y$ are Banach spaces and $F \in \Gamma(X \times Y)$. Then

${ }^{i c}\{A x-y \mid(x, y) \in \operatorname{dom} \partial F\}=\operatorname{ri}\{A x-y \mid(x, y) \in \operatorname{dom} \partial F\}={ }^{i c} D=\operatorname{ri} D$,

while if one of the above sets is nonempty then

$$
\overline{\{A x-y \mid(x, y) \in \operatorname{dom} \partial F\}}=\overline{i c\{A x-y \mid(x, y) \in \operatorname{dom} \partial F\}}=\bar{D} .
$$

In particular, if ${ }^{i c} D \neq \emptyset$ then the sets ${ }^{i c}\{A x-y \mid(x, y) \in \operatorname{dom} \partial F\}$ and $\overline{\{A x-y \mid(x, y) \in \operatorname{dom} \partial F\}}$ are convex.

PROOF. The first part follows from Proposition 4 taking $\Phi(x, y)=F(x, A x-y)$. Suppose that $X, Y$ are Banach spaces, $F \in \Gamma(X \times Y)$ and consider $E=|A x-y|$ $(x, y) \in \operatorname{dom} \partial F$ ]. By Brøndsted-Rockafellar theorem (see [30, Theorem 3.18]) we have that $\operatorname{dom} F \subset \overline{\operatorname{dom} \partial F}$. Hence

$$
E \subset D \subset\{A x-y \mid(x, y) \in \overline{\operatorname{dom} \partial F}\} .
$$

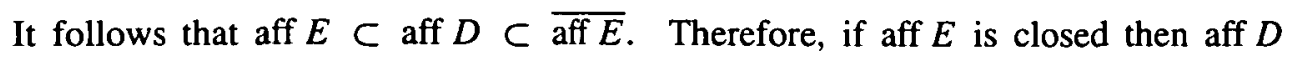
is closed, and the above relation shows that ${ }^{i c} E \subset{ }^{i c} D(=$ ri $D)$. Let us show 
the converse inclusion. Let $y_{0} \in{ }^{i c} D$ and consider the function $F_{0} \in \Gamma(X \times Y)$, $F_{0}(x, y)=F\left(x, y-y_{0}\right)$. Of course, $\operatorname{dom} F_{0}=\operatorname{dom} F+\left(0, y_{0}\right)$ and $\operatorname{dom} \partial F_{0}=$ $\operatorname{dom} \partial F+\left(0, y_{0}\right)$. Therefore $0 \in{ }^{i c}\left(\operatorname{Pr}_{Y}\left(\operatorname{dom} F_{0}\right)\right)$. Taking $\varphi_{0}(x)=F_{0}(x, A x)$, $\varphi_{0} \in \Gamma(X)$. Hence dom $\partial \varphi_{0} \neq \emptyset$, which shows, by the preceding theorem, that there exists $x$ such that $\partial F_{0}(x, A x)=\partial F\left(x, A x-y_{0}\right) \neq \emptyset$. Therefore $y_{0} \in E$. So we obtained that ${ }^{i} D={ }^{i c} D \subset E \subset D$, which shows that aff $E=$ aff $D$ and ${ }^{i} D \subset{ }^{i} E$. It follows that ${ }^{i c} E={ }^{i c} D=$ rint $E$.

Suppose that ${ }^{i c} D \neq \emptyset$ (for example). From what was proved above, we have that

$$
\text { rint } D={ }^{i} D={ }^{i c} D=\operatorname{rint} E={ }^{i} E={ }^{i c} E \subset E \subset D \subset \bar{E} \text {. }
$$

Hence $\bar{E}=\overline{{ }^{i c} E}=\bar{D}$. The conclusion follows.

REMARK 6. Taking into account the preceding proposition, for $X, Y$ Banach spaces and $F \in \Gamma(X \times Y)$, we may add to the sufficient conditions in Theorem 6 the following conditions:

$$
\begin{aligned}
& 0 \in \operatorname{ri}\{A x-y \mid(x, y) \in \operatorname{dom} \partial F\}, \\
& 0 \in^{i c}\{A x-y \mid(x, y) \in \operatorname{dom} \partial F\}, \\
& 0 \in^{i c}\{A x-y \mid(x, y) \in \operatorname{conv}(\operatorname{dom} \partial F)\},
\end{aligned}
$$

and

$$
Y_{0}=\operatorname{cone}\{A x-y \mid(x, y) \in \operatorname{dom} \partial F\} \text { is a closed linear space. }
$$

Indeed, the first three conditions are, evidently (using the preceding proposition), equivalent to $0 \in{ }^{i c}\{A x-y \mid(x, y) \in \operatorname{dom} F\}$. In the fourth case $Y_{0}=\operatorname{aff}\{A x-y \mid$ $(x, y) \in \operatorname{dom} \partial F\}$, and therefore $0 \in{ }^{i c}\{A x-y \mid(x, y) \in \operatorname{dom} F\}$.

\section{Fenchel-Rockafellar duality}

Consider now the case when $F$ from the preceding section has separated variables.

THEOREM 8. Let $f \in \Lambda(X), g \in \Lambda(Y)$ and $A \in \mathscr{L}(X, Y)$. Assume that $\operatorname{dom} f \cap$ $A^{-1}(\operatorname{dom} g) \neq \emptyset$ and let $Y_{0}=\operatorname{span}(A(\operatorname{dom} f)-\operatorname{dom} g)$. Consider $\varphi_{2} \in \Lambda(X)$, $\varphi_{2}(x)=f(x)+g(A x)$. If one of the following conditions holds:

(i) there exist $\lambda_{0} \in \mathbf{R}, B \in \mathscr{B}_{X}$ and $V_{0} \in \mathscr{V}_{Y_{0}}$ such that

$$
V_{0} \subset A\left(\left[f \leq \lambda_{0}\right] \cap B\right)-\left[g \leq \lambda_{0}\right]
$$


(ii) for every $U \in \mathscr{V}_{X}$ there exist $\lambda>0$ and $V \in \mathscr{V}_{Y_{0}}$ such that

$$
V \subset A([f \leq \lambda] \cap \lambda U)-[g \leq \lambda]
$$

(iii) $\operatorname{dim} Y_{0}<\infty$ and $0 \in \in^{i}(A(\operatorname{dom} f)-\operatorname{dom} g)$;

(iv) there exists $x_{0} \in \operatorname{dom} f \cap A^{-1}(\operatorname{dom} g)$ such that $g$ is continuous at $A x_{0}$;

(v) $X$ is a Fréchet space, $f, g$ are l.s.c. and $0 \in{ }^{i b}(A(\operatorname{dom} f)-\operatorname{dom} g)$;

(vi) $X, Y$ are metrizable, $f, g$ have proper conjugates, $[(f, g$ are cs-closed and $\operatorname{gr} A$ is cs-complete) or $f, g$ are cs-complete] and $0 \in{ }^{i b}(A(\operatorname{dom} f)-\operatorname{dom} g)$;

(vii) $X, Y$ are Fréchet spaces, $f, g$ are l.s.c. and $0 \in{ }^{i c}(A(\operatorname{dom} f)-\operatorname{dom} g)$; (viii) $Y=\mathbf{R}^{n}$, qri(dom $\left.f\right) \neq \emptyset$ and $A(\operatorname{qri}(\operatorname{dom} f)) \cap{ }^{i} \operatorname{dom} g \neq \emptyset$,

then

$$
\begin{aligned}
\inf \{f(x)+g(A x) \mid x \in X\} & =\max \left\{-f^{*}\left(A^{*} y^{*}\right)-g^{*}\left(-y^{*}\right) \mid y^{*} \in Y^{*}\right\}, \\
\forall x^{*} \in X^{*}: \varphi_{2}^{*}\left(x^{*}\right) & =\min \left\{f^{*}\left(x^{*}-A^{*} y^{*}\right)+g^{*}\left(y^{*}\right) \mid y^{*} \in Y^{*}\right\}, \\
\forall x \in \operatorname{dom} \varphi_{2}, \varepsilon \geq 0: \partial_{\varepsilon} \varphi_{2}(x) & =\bigcup_{\varepsilon_{1}, \varepsilon_{2} \geq 0, \varepsilon_{1}+\varepsilon_{2}=\varepsilon}\left(\partial_{\varepsilon_{1}} f(x)+A^{*}\left(\partial_{\varepsilon_{2}} g(A x)\right)\right) .
\end{aligned}
$$

PROOF. Consider $F: X \times Y \rightarrow \overline{\mathbf{R}}, F(x, y)=f(x)+g(y)$. It is obvious that $F$ is l.s.c. if and only if $f, g$ are l.s.c., $\operatorname{dom} F=\operatorname{dom} f \times \operatorname{dom} g, F^{*}\left(x^{*}, y^{*}\right)=$ $f^{*}\left(x^{*}\right)+g^{*}\left(y^{*}\right)$ and $\partial_{\varepsilon} F(x, y)=\bigcup_{\varepsilon_{1}, \varepsilon_{2} \geq 0, \varepsilon_{1}+\varepsilon_{2}=\varepsilon} \partial_{\varepsilon_{1}} f(x) \times \partial_{\varepsilon_{2}} g(y)$. It is easy to see that if one of the conditions (i)-(vii) holds then the corresponding condition from Theorem 6 is verified. If (viii) holds, using the properties of the intrinsic core in finite-dimensional spaces and [10, Proposition 2.10] (recalled in Section 2), we have

$$
{ }^{i}(A(\operatorname{dom} f)-\operatorname{dom} g)={ }^{i}(A(\operatorname{dom} f))-{ }^{i}(\operatorname{dom} g)=A(\operatorname{qri}(\operatorname{dom} f))-{ }^{i}(\operatorname{dom} g),
$$

and so (iii) holds too. The conclusions follow.

REMARK 7. As in Theorem 6, we have that (iii) $\Rightarrow$ (i) $\Rightarrow$ (ii), (iv) $\Rightarrow$ (i), (vii) $\Rightarrow$ (v) $\Rightarrow$ (ii), (vii) $\Rightarrow$ (vi), and of course, as mentioned in the proof, (viii) $\Rightarrow$ (iii).

Note that conditions (i) and (ii) of Theorem 6 for $F(x, y)=f(x)+g(y)$ imply conditions (i) and (ii) of Theorem 8, respectively, if $f$ and $g$ have proper conjugates. Indeed, let $f(x) \geq\left\langle x, x^{*}\right\rangle+\alpha$ for $x \in X$ and $g(y) \geq\left\langle y, y^{*}\right\rangle+\beta$ for $y \in Y$, where $x^{*} \in X^{*}, y^{*} \in Y^{*}, \alpha, \beta \in \mathbf{R}$ are fixed. Take $U_{0}=\left\{x \in X||\left\langle x, x^{*}\right\rangle \mid \leq 1\right\}$ and $V_{0}=\left\{y \in Y||\left\langle y, y^{*}\right\rangle \mid \leq 1\right\}$. Assume that condition (ii) of Theorem 6 holds. Let $U \in \mathscr{V}_{X}$, and take $U^{\prime}=U \cap U_{0}$; there exist $\lambda^{\prime}>0$ and $V^{\prime} \in \mathscr{Y}_{Y_{0}}$ such that

$$
\{0\} \times V^{\prime} \subset\left\{(x, A x) \mid x \in \lambda^{\prime} U^{\prime}\right\}-\left[F \leq \lambda^{\prime}\right] .
$$


Let $y \in V_{0} \cap V^{\prime}$; there exist $x \in \lambda^{\prime} U^{\prime}, u \in \operatorname{dom} f, v \in \operatorname{dom} g$ such that $f(u)+g(v) \leq$ $\lambda^{\prime}$ and $(0, y)=(x, A x)-(u, v)$. Hence $x=u$ and $y=A x-v$. It follows that

$$
g(v) \leq \lambda^{\prime}-f(x) \leq \lambda^{\prime}-\left\langle x, x^{*}\right\rangle-\alpha \leq \lambda^{\prime}+1-\alpha,
$$

and

$$
f(x) \leq \lambda^{\prime}-g(v) \leq \lambda^{\prime}-\left\langle v, y^{*}\right\rangle-\beta \leq \lambda^{\prime}+1-\beta .
$$

Taking $\lambda=\max \left\{\lambda^{\prime}, \lambda^{\prime}+1-\alpha, \lambda^{\prime}+1-\beta\right\}$ and $V=V_{0} \cap V^{\prime} \in \mathscr{V}_{Y_{0}}$, we have that (ii) of Theorem 8 holds.

In a similar way one obtains that (i) of Theorem 8 holds if (i) of Theorem 6 does.

REMARK 8. All the statements of Proposition 7 hold for the corresponding hypotheses; in this case $D=A(\operatorname{dom} f)-\operatorname{dom} g, F$ 1.s.c. is replaced by $f, g$ 1.s.c., $F$ cs-closed (cs-complete) is replaced by $f, g$ have proper conjugates and are cs-closed (cs-complete) and dom $\partial F$ is replaced by dom $\partial f \times \operatorname{dom} \partial g$.

REMARK 9. The conclusions of Theorem 8 (with $\varepsilon=0$ ) are stated by Rockafellar [34] using condition (iv). Conditions (iv) and (vii) [for $X, Y$ Banach spaces, $X$ reflexive and $Y_{0}=Y$ ] are used by Rockafellar [36, Example 11'] for the FenchelRockafellar duality formula (11). Condition (iv) is used also in [15, Remark 3.4.2], [5, Theorem 3.2.4] and [41, Proposition 3.2] [with $\mathbf{R}$ replaced by an ordered space] for (11). The same formula is obtained under a condition stronger than vii) by Aubin [3, Theorem 14.1.3] [for $X, Y$ reflexive Banach spaces and $0 \in \operatorname{int}(A(\operatorname{dom} f)-\operatorname{dom} g)$ ], by Borwein [8, Theorem 8.1] [for $Y_{0}=Y$ ] (see also [48]), by Attouch and Brézis [1, Corollary 2.3] [for $X, Y$ Banach spaces and $Y_{0}=Y$ ], by Cominetti [14, Theorem 2] [for $X, Y$ Banach spaces and $Y_{0}=Y$ ], by Azé [4, Theorem 3.2] [for $X, Y$ Banach spaces] and by Gowda and Teboulle [16, Theorem 3.5 and p. 931]. Rodrigues and Simons [38, Theorem 6] and Rodrigues [37, Theorem 2.5, Corollary 3.1] use condition (vii), while Simons [39, Theorem 5] uses a stronger form of (vi) $[f, g$ cs-convex, $X, Y$ Fréchet spaces and $\left.0 \in{ }^{i c}(A(\operatorname{dom} f)-\operatorname{dom} g)\right]$ to obtain (12) and (13) (with $\left.\varepsilon=0\right)$; Combari, Laghdir and Thibault [12, Corollary 4.16] use conditions (iv) and (vii), while the same authors in [13, Corollary 4.6] use (ii) for the same conclusions. Conditions (i), (iii), (iv) and (vii) [for $X, Y$ Banach spaces] are used in [51, Theorem 2.7.3].

In [36, p. 50], it is suggested that (11) (with $x \in D(A)$ and $y^{*} \in D\left(A^{*}\right)$ ) is also valid if $X, Y$ are reflexive Banach spaces, $f \in \Gamma(X), g \in \Gamma(Y), A: D(A) \subset X \rightarrow Y$ is a densely defined linear operator with closed graph and $0 \in(A(\operatorname{dom} f)-\operatorname{dom} g)^{i}$. Applying [8, Theorem 8.1], (11) would be true even if $X, Y$ are Fréchet spaces. The following example shows that this is not true. 
Let $X=Y=\ell^{2}$ and $A: D(A) \subset \ell^{2} \rightarrow \ell^{2}, A(x)=\left(x_{1}, 2 x_{2}, \ldots, n x_{n}, \ldots\right)$, where $D(A)=\left\{x=\left(x_{1}, x_{2}, \ldots\right) \in \ell^{2} \mid\left(x_{1}, 2 x_{2}, \ldots, n x_{n}, \ldots\right) \in \ell^{2}\right\} ;$ it is easy to see that $A$ is a densely defined linear operator with closed graph and $A^{*}=A$. Consider also $\bar{x}=(1,1 / 2, \ldots, 1 / n, \ldots) \in \ell^{2} \backslash D(A), f \in \Gamma\left(\ell^{2}\right), f(x)=t$ if $x=t \bar{x},=\infty$ otherwise, and $g=0$. It is clear that all the above conditions are verified, but

$$
\inf \{f(x)+g(A x) \mid x \in D(A)\}=\inf \{f(x) \mid x \in D(A)\}=f(0)=0,
$$

while

$$
\sup \left\{-f^{*}\left(A^{*} y^{*}\right)-g^{*}\left(-y^{*}\right) \mid y^{*} \in D\left(A^{*}\right)\right\}=-f^{*}(0),
$$

because dom $g^{*}=\{0\}$. As $-f^{*}(0)=\inf \left\{f(x) \mid x \in \ell^{2}\right\}=-\infty$, the FenchelRockafellar duality formula does not hold in this case. ${ }^{3}$

When $f=0$ it is possible to establish similar results to those in Theorem 8 for $A$ a densely defined linear operator with closed graph. The next theorem is stated even for $A$ replaced by a convex process $\mathscr{A}$. We recall that for the convex process $\mathscr{A} \subset X \times Y$ (that is, $\mathscr{A}$ is a convex cone), its adjoint is the (closed) convex process $\mathscr{A}^{*}=\left\{\left(y^{*}, x^{*}\right) \in Y^{*} \times X^{*} \mid\left(-x^{*}, y^{*}\right) \in \mathscr{A}^{+}\right\}$.

THEOREM 9. Let $g \in \Lambda(Y)$ and $\mathscr{A} \subset X \times Y$ a convex process. Assume that $0 \in D$, where $D=\mathscr{A}(X)-\operatorname{dom} g$, and consider $Y_{0}=$ span $D$. If one of the following conditions holds:

(i) for every $U \in \mathscr{V}_{X}$ there exist $\lambda>0$ and $V \in \mathscr{V}_{Y_{0}}$ such that $V \subset[g \leq$ ג] $-\mathscr{A}(\lambda U)$;

(ii) there exist $\lambda_{0} \in \mathbf{R}, B \in \mathscr{B}_{X}$ and $V_{0} \in \mathscr{V}_{Y_{0}}$ such that $V \subset[g \leq \lambda]-\mathscr{A}(B)$;

(iii) $\operatorname{dim} Y_{0}<\infty$ and $0 \in{ }^{i} D$;

(iv) there exists $x_{0} \in \mathscr{A}^{-1}(\mathrm{dom} g)$ such that $g$ is continuous at some point in $\mathscr{A}\left(x_{0}\right)$;

(v) $X, Y$ are metrizable, $g$ has proper conjugate, [( $g$ is cs-closed and $\mathscr{A}$ is cscomplete) or ( $g$ is cs-complete and $\mathscr{A}$ verify $(\mathrm{Hx}))]$ and $0 \in{ }^{i b} D$;

(vi) $X, Y$ are Fréchet spaces, $g$ is l.s.c., $\mathscr{A}$ is closed and $0 \in{ }^{i b} D$, and $\varphi_{3}: X \rightarrow \overline{\mathbf{R}}, \varphi_{3}(x)=\inf \{g(y) \mid y \in \mathscr{A}(x)\}$, then

$$
\forall x^{*} \in X^{*}: \varphi_{3}^{*}\left(x^{*}\right)=\min \left\{g^{*}\left(y^{*}\right) \mid y^{*} \in \mathscr{A}\left(x^{*}\right)\right\} .
$$

Moreover, if $\bar{x} \in \operatorname{dom} \varphi_{3}=\mathscr{A}^{-1}(\operatorname{dom} g)$ is such that $\varphi_{3}(\bar{x})=g(\bar{y})$ with $\bar{y} \in \mathscr{A}(\bar{x})$, and $\varepsilon \geq 0$ then $\partial_{\varepsilon} \varphi_{3}(\bar{x}) \subset \mathscr{A}^{*}\left(\partial_{\varepsilon} g(\bar{y})\right.$ ) (with equality if $\mathscr{A}$ is a linear subspace).

\footnotetext{
${ }^{3}$ As observed by the referee, the preceding counterexample works for an arbitrary densely defined and closed linear operator $A: D(A) \subset X \rightarrow Y, g=0$ and $f$ defined similarly for $\bar{x} \in X \backslash D(A)$.
} 
PROOF. Taking $F(x, y)=g(y)$ in Theorem 5, perturbed with $x^{*} \in X^{*}$, one obtains the expression of $\varphi_{3}^{*}\left(x^{*}\right)$. For obtaining $\partial_{\varepsilon} \varphi_{3}(\bar{x})$ one uses the standard argument.

Note that (iii) $\Rightarrow$ (ii) $\Rightarrow$ (i), (iv) $\Rightarrow$ (ii), (vi) $\Rightarrow$ (i) and (vi) $\Rightarrow$ (v).

Generally, the conclusions of the preceding theorem, for $\mathscr{A}=$ gr $A$ with $A \in$ $\mathscr{L}(X, Y)$, is obtained applying Theorem 8 taking $f=0$ (see Remark 9 for references), or directly using condition (iv) (in infinite-dimensional spaces) and condition (iii) in finite-dimensional spaces. In the next remark we do not refer to that situations. Note that in [36] and [17] are used other conditions, too.

REMARK 10. The formula for $\varphi_{3}^{*}\left(x^{*}\right)$ under condition (v) is obtained in [50, Proposition 7]. Let $\mathscr{A}=\mathrm{gr} A$ with $A$ a densely defined linear operator with closed graph. Rockafellar [36, Theorem 19(c)] obtains the results (with $\varepsilon=0$ ) under condition (vi) [for $X, Y$ Banach spaces, $X$ reflexive and $Y_{0}=Y$ ], while Hirriart-Urruty [17, Theorem 2.2] obtains them under (vi) [for $X, Y$ Banach spaces and $Y_{0}=Y$ ]; Azé [4, Theorem 2.1] uses condition (ii) to obtain $\varphi_{3}^{*}\left(x^{*}\right)$ [for $X, Y$ normed spaces and $Y_{0}=Y$ ]. For $A \in \mathscr{L}(X, Y)$, condition (vi) is used by Penot [29, Theorem 3.7] to obtain $\partial \varphi_{3}$ [for $X, Y$ Banach spaces and $Y_{0}=Y$ ], Zălinescu [46], [47, Theorem 4 (8)] [for $Y_{0}=Y$ ], by Rodrigues [37, Theorem 2.3] to obtain $\varphi_{3}^{*}\left(x^{*}\right)$ [for ${ }^{i b} D$ replaced by ${ }^{i c} D$ ]. When $A: X \rightarrow Y$ is linear with complete graph the conclusions of the theorem (with $\varepsilon=0$ ) are obtained under variants of $(v)$ by Rodrigues and Simons [38, Corollary 4] [for $Y$ a Fréchet space, $g$ l.s.c. and ${ }^{i b} D$ replaced by ${ }^{i c} D$ ] and by Simons [39, Theorem 4] [for $g$ cs-convex].

\section{Fenchel duality formula}

In this section we are interested in duality, conjugate and $\varepsilon$-subdifferential for the sum of two convex functions. The main result is the following.

THEOREM 10. Let $f, g \in \Lambda(X)$. Assume that $\operatorname{dom} f \cap \operatorname{dom} g \neq \emptyset$ and let $X_{0}=$ span $(\operatorname{dom} f-\operatorname{dom} g)$. If one of the following conditions holds:

(i) there exist $\lambda_{0} \in \mathbf{R}, B \in \mathscr{B}_{X}$ and $V_{0} \in \mathscr{V}_{X_{0}}$ such that

$$
V_{0} \subset\left[f \leq \lambda_{0}\right] \cap B-\left[g \leq \lambda_{0}\right] ;
$$

(ii) for every $U \in \mathscr{V}_{X}$ there exist $\lambda>0$ and $V \in \mathscr{V}_{X_{0}}$ such that

$$
V \subset[f \leq \lambda] \cap \lambda U-[g \leq \lambda]
$$

(iii) $\operatorname{dim} X_{0}<\infty$ and $0 \in{ }^{i}(\operatorname{dom} f-\operatorname{dom} g)$;

(iv) there exists $x_{0} \in \operatorname{dom} f \cap \operatorname{dom} g$ such that $g$ is continuous at $x_{0}$; 
(v) $X$ is a Fréchet space, $f, g \in \Gamma(X)$ and $0 \in{ }^{i b}(\operatorname{dom} f-\operatorname{dom} g)$;

(vi) $X$ is metrizable, $f, g$ have proper conjugates, $f$ is cs-closed, $g$ is cs-complete and $0 \in{ }^{i b}(\operatorname{dom} f-\operatorname{dom} g)$;

(vii) $X$ is a Fréchet space, $f, g \in \Gamma(X)$ and $0 \in{ }^{i c}(\operatorname{dom} f-\operatorname{dom} g)$;

(viii) $X$ is a Banach space, $f, g \in \Gamma(X)$ and $0 \in{ }^{i c}(\operatorname{dom} \partial f-\operatorname{dom} \partial g)$;

(ix) $g$ is quasi-continuous and $\operatorname{dom} f$ and $\operatorname{dom} g$ are united,

then

$$
\begin{aligned}
& \inf \{f(x)+g(x) \mid x \in X\}=\max \left\{-f^{*}\left(x^{*}\right)-g^{*}\left(-x^{*}\right) \mid x^{*} \in X^{*}\right\} \\
& \forall x^{*} \in X^{*}:(f+g)^{*}\left(x^{*}\right)=\min \left\{f^{*}\left(x^{*}-y^{*}\right)+g^{*}\left(y^{*}\right) \mid y^{*} \in X^{*}\right\},
\end{aligned}
$$

and

$$
\forall x \in \operatorname{dom} f \cap \operatorname{dom} g, \varepsilon \geq 0: \partial_{\varepsilon}(f+g)(x)=\bigcup_{\varepsilon_{1}, \varepsilon_{2} \geq 0, \varepsilon_{1}+\varepsilon_{2}=\varepsilon}\left(\partial_{\varepsilon_{1}} f(x)+\partial_{\varepsilon_{2}} g(x)\right) .
$$

PROOF. Taking $A=\mathrm{Id}_{X}$, the conclusion follows from Theorem 8 under conditions (i)-(v) and (vii). When $X$ is a Banach space conditions (vii) and (viii) are equivalent by Proposition 11 below. If (vi) or (ix) holds, taking $Y=X$ and $\Phi(x, y)=f(x)+$ $g(x-y)$, condition (v) or (vii) (respectively) of Theorem 3 is verified.

Note that (iii) $\Rightarrow$ (i) $\Rightarrow$ (ii), (iv) $\Rightarrow$ (i), (vii) $\Rightarrow$ (v) $\Rightarrow$ (ii) and (v) $\Rightarrow$ (vi).

Generally, the conclusions of the preceding theorem are obtained using condition (iv) (in infinite-dimensional spaces; see $[24,27,33]$, etc.) and condition (iii) in finitedimensional spaces. In the next remark we do not refer to that situations. Note that in [36] and [17] are used other conditions, too; see also [48, Proposition 1].

Ponstein [31, Theorem 3.14.20] obtains (14) using the condition: $X$ is a Banach space, $\operatorname{ri}(\operatorname{dom} f) \cap \operatorname{ri}(\operatorname{dom} g) \neq \emptyset$, whence $X_{1}=\operatorname{aff}(\operatorname{dom} f)$ and $X_{2}=\operatorname{aff}(\operatorname{dom} g)$ are closed, $\left.f\right|_{X_{1}},\left.g\right|_{X_{2}}$ are (upper semi-) continuous on $\operatorname{ri}(\operatorname{dom} f$ ) and $\operatorname{ri}(\operatorname{dom} g$ ), respectively, and $X_{1}+X_{2}$ is closed. This condition is stronger than (ii) [or, equivalently, (i)].

Indeed, let $x_{0} \in \operatorname{ri}(\operatorname{dom} f) \cap \operatorname{ri}(\operatorname{dom} g)$. Using a standard argument, one may assume that $x_{0}=0$, and so $X_{1}$ and $X_{2}$ are closed linear subspaces, as well as $X_{0}=X_{1}-X_{2}=\operatorname{span}(\operatorname{dom} f-\operatorname{dom} g)$. By hypothesis, there exist $\rho, \lambda>0$ such that

$$
\rho U_{X} \cap X_{1} \subset[f \leq \lambda], \quad \rho U_{X} \cap X_{2} \subset[g \leq \lambda] .
$$

As $T: X_{1} \times X_{2} \rightarrow X_{0}, T\left(x_{1}, x_{2}\right)=x_{1}+x_{2}$, is linear and continuous and $X_{1} \times X_{2}, X_{0}$ are Banach spaces, there exists $\eta>0$ such that $X_{0} \cap \eta U_{X} \subset X_{1} \cap \rho U_{X}-X_{2} \cap \rho U_{X}$. It follows that $X_{0} \cap \eta U_{X} \subset[f \leq \lambda] \cap \rho U_{X}-[g \leq \lambda] \cap \rho U_{X}$. Therefore (ii) holds. 
REMARK 11. Condition (ix) is used by Joly and Laurent [21, Proposition 2.1.6] (see also [24, Proposition 7.8.5]) for (14); it seems that (ix) is used by Joly [20] for (15) (see [26]). Note also that Mentagui [26, Proposition 3.1] shows that $0 \in{ }^{i c}(\operatorname{dom} f-\operatorname{dom} g$ ) if $X$ is a Banach space and (ix) holds. Condition (vii) is used by Rockafellar [36, Theorem 20 (c)] (under an equivalent form) [for $X$ a reflexive Banach space and $X_{0}=$ $X$ ], Penot [29, Theorem 3.6], Zălinescu [47, Theorem 4 (15)] [for $X_{0}=X$ ], Attouch and Brézis [1, Theorem 1.1 and Corollary 3.1] [for $X$ a Banach space], Zălinescu [50, Corollary 4], Rodrigues and Simons [38, Corollary 7], Combari, Laghdir and Thibault [12, Corollary 4.16]; a stronger form of (vi) [ $X$ a Fréchet space, $f, g$ cs-closed and $0 \in{ }^{i c}(\operatorname{dom} f-\operatorname{dom} g)$ ] is used by Simons [39, Corollary 6], condition (vi) is used in [45, Corollary 1], condition (viii) is used in [2, Theorem 4.2] [for $X$ a reflexive Banach space], while condition (ii) is introduced by Combari, Laghdir and Thibault [13, Corollary 4.7] for (15) and (16) with $\varepsilon=0$; Jeyakumar [18, Lemma 3.1] uses (vii) for the Fenchel duality formula (14). Condition (i) was first used by Azé [4, Corollary 2.2] [for $X$ a normed space under the form: there exist $s, r, \lambda>0$ such that $\left.s U_{X} \subset[f \leq \lambda] \cap r U_{X}-[g \leq \lambda] \cap r U_{X}\right]$ for obtaining (15). Conditions (i) and (vii) [for $X$ Banach spaces] are used in [51, Theorem 2.7.3].

Proposition 11. Let $f, g \in \Lambda(X)$ and take $D=\operatorname{dom} f-\operatorname{dom} g$. If $\operatorname{dim}(\operatorname{span} D)$ $<\infty$ then $^{i} D=$ rint $D$, while if $X$ is metrizable, $f, g$ have proper conjugates, $f$ is $c s$-closed, $g$ is cs-complete and ${ }^{i b} D \neq \emptyset$ then ${ }^{i b} D=$ rint $D$.

Suppose that $X$ is a Banach space and $f, g \in \Gamma(X)$. Then for every $x \in{ }^{i b} D$ there exist $\eta, \lambda>0$ such that

$$
\left(x+\eta U_{X}\right) \cap \text { aff } D \subset[f \leq \lambda] \cap \lambda U_{X}-[g \leq \lambda] \cap \lambda U_{X} .
$$

Moreover, taking $E=\operatorname{dom} \partial f-\operatorname{dom} \partial g$, we have

$$
{ }^{i c} E=\operatorname{ri} E={ }^{i c}(\operatorname{conv} E)=\operatorname{ri}(\operatorname{conv} E)={ }^{i c} D=\operatorname{ri} D,
$$

and, if ${ }^{i c} D$ is nonempty then $\overline{{ }^{i c} E}=\bar{E}=\bar{D}$.

PROOF. The first part follows from Proposition 4 taking $Y=X$ and $\Phi(x, y)=$ $f(x)+g(x-y)$.

Suppose that $X$ is a Banach space and $f, g \in \Gamma(X)$ and take $x \in{ }^{i b} D$. Replacing $f$ by $\widetilde{f}, \widetilde{f}(u)=f(u+x)$, we may suppose that $x=0$. It follows that condition (vii) of Theorem 10 holds, and, as noted after its proof, condition (i) is verified. Therefore there exist $\eta>0, \lambda_{0} \in \mathbf{R}$ and $B \in \mathscr{B}_{X}$ such that

$$
\eta U_{x} \cap \text { aff } D \subset\left[f \leq \lambda_{0}\right] \cap B-\left[g \leq \lambda_{0}\right]
$$


Taking $\lambda^{\prime}>\max \left\{\lambda_{0}, 0\right\}$ such that $B \subset \lambda^{\prime} U_{X}$ and $\lambda=\lambda^{\prime}+\eta$ we obtain that

$$
\begin{gathered}
\eta U_{X} \cap \text { aff } D \subset\left[f \leq \lambda_{0}\right] \cap B-\left[g \leq \lambda_{0}\right] \cap\left(B+\eta U_{X}\right) \\
\subset[f \leq \lambda] \cap \lambda U_{X}-[g \leq \lambda] \cap \lambda U_{X} .
\end{gathered}
$$

The rest of the conclusion follows from Proposition 7 taking $F(x, y)=f(x)+g(y)$ and $A=\mathrm{Id}_{X}$.

REMARK 12. Attouch and Théra [2, Theorem 4.10] obtain the weaker formula int $(\operatorname{dom} f-\operatorname{dom} g)=\operatorname{int}(\operatorname{dom} \partial f-\operatorname{dom} \partial g)$ when $X$ is a reflexive Banach space, while Simons [40, Theorem 36] obtains (practically) the same formula for $X$ only a Banach space. Note the particular case $x=0 \in D^{i}$ (whence aff $D=X$ ) of the preceding proposition. In this situation from (17) one obtains Lemma 1, Theorem 3 and Corollary 4 of Simons [40], and Lemma 3.4 of Azé [4].

\section{Conjugates and subdifferentials of composed convex functions}

In this section $Y$ is ordered by a convex cone $Q \subset Y$ (for $y_{1}, y_{2} \in Y, y_{1} \leq y_{2}$ if and only if $y_{2}-y_{1} \in Q$ ). We adjoin to $Y$ a greatest element $\infty$ getting $Y^{\bullet}=Y \cup\{\infty\}$ and extend the algebraic operations and the order on $Y^{\bullet}$ as usually. Consider $H: X \rightarrow Y^{\bullet}$; dom $H=\{x \in X \mid H(x) \in Y\}$, epi $H=\{(x, y) \in X \times Y \mid H(x) \leq y\}$. $H$ is convex if epi $H$ is convex (that is, $H\left(\lambda x_{1}+(1-\lambda) x_{2}\right) \leq \lambda H\left(x_{1}\right)+(1-\lambda) H\left(x_{2}\right)$ for all $\lambda \in(0,1)$ and $\left.x_{1}, x_{2} \in X\right)$. Let $g: Y \rightarrow \overline{\mathbf{R}}$. We say that $g$ is increasing on $E \subset Y$ if $y_{1}, y_{2} \in E$ and $y_{1} \leq y_{2}$ imply $g\left(y_{1}\right) \leq g\left(y_{2}\right) ; g$ is increasing if $g$ is increasing on the whole space $Y$. For $g$ and $H$ as above, we define $g \circ H$ by $(g \circ H)(x)=g(H(x))$ if $x \in \operatorname{dom} H,=\infty$ otherwise (which amounts to say that $g(\infty)=\infty$ ).

In the next theorem we give formulae for the conjugate and $\varepsilon$-subdifferential of $f+g \circ H$.

THEOREM 12. Let $Y$ be ordered by the convex cone $Q, f \in \Lambda(X), H: X \rightarrow Y^{\bullet}$ be convex and $g \in \Lambda(Y)$ be increasing on $H(\operatorname{dom} H)+Q$. Then $\varphi_{4}=f+g \circ H$ is convex. Assume that $0 \in D$, where $D=H(\operatorname{dom} H \cap \operatorname{dom} f)-\operatorname{dom} g+Q$, and consider $Y_{0}=\operatorname{span} D$. If one of the following conditions holds:

(i) for every $U \in \mathscr{V}_{X}$ there exist $\lambda>0$ and $V \in \mathscr{Y}_{Y_{0}}$ such that

$$
V \subset H(\lambda U \cap[f \leq \lambda] \cap \operatorname{dom} H)-[g \leq \lambda]+Q
$$

(ii) there exist $\lambda_{0} \in \mathbf{R}, B \in \mathscr{B}_{X}$ and $V_{0} \in \mathscr{V}_{Y_{0}}$ such that

$$
V_{0} \subset H\left(B \cap\left[f \leq \lambda_{0}\right] \cap \operatorname{dom} H\right)-\left[g \leq \lambda_{0}\right]+Q ;
$$


(iii) $\operatorname{dim} Y_{0}<\infty$ and $0 \in{ }^{i} D$;

(iv) there exists $x_{0} \in \operatorname{dom} f \cap H^{-1}(\operatorname{dom} g)$ such that $g$ is continuous at $H\left(x_{0}\right)$;

(v) $X, Y$ are metrizable, $f, g$ have proper conjugates, $[(f, g$ are cs-closed and epi $H$ is cs-complete) or ( $f, g$ are cs-complete and epi $H$ is cs-closed)] and $0 \in{ }^{i b} D$;

(vi) $X, Y$ are Fréchet spaces, $f, g$ are l.s.c. and $0 \in{ }^{i b} D$, then

$$
\begin{aligned}
& \forall x^{*} \in X^{*}: \varphi_{4}^{*}\left(x^{*}\right)=\min \left\{\left(f+y^{*} \circ H\right)^{*}\left(x^{*}\right)+g^{*}\left(y^{*}\right) \mid y^{*} \in Q^{+}\right\}, \\
& \forall \varepsilon \geq 0, x \in \operatorname{dom} \varphi_{4}: \partial_{\varepsilon} \varphi_{4}(x) \\
& \quad=\bigcup\left\{\partial_{\varepsilon_{1}}\left(f+y^{*} \circ H\right)(x) \mid y^{*} \in \partial_{\varepsilon_{2}} g(H(x)) \cap Q^{+}, \varepsilon_{1}+\varepsilon_{2}=\varepsilon\right\} .
\end{aligned}
$$

PROOF. Let $F(x, y)=f(x)+g(y)$ and $\mathscr{A}=$ epi $H ; F \in \Lambda(X \times Y)$ and $\mathscr{A}$ is convex. Since $g$ is increasing on $H(\operatorname{dom} H)+Q$, it follows that $\varphi_{4}(x)=\inf \{F(x, y)+$ $\left.I_{\text {epi } H}(x, y) \mid y \in Y\right\}$ for every $x \in X$. Hence $\varphi_{4}$ is the marginal function associated to the convex function $F+I_{\infty}$; hence $\varphi_{4}$ is convex.

If one of the conditions (i)-(vi) is verified, then $F$ and $\mathscr{A}$ satisfy the corresponding condition of Theorem 5 . As noticed after that theorem, also the perturbed function $\widetilde{F}, \widetilde{F}(x, y)=F(x, y)-\left\langle x, x^{*}\right\rangle$, satisfies the same condition. Therefore there exists $z^{*} \in Y^{*}$ such that

$$
\inf _{(x, y) \in \text { epi } H}\left(f(x)+g(y)-\left\langle x, x^{*}\right\rangle\right)=\inf _{(x, y+z) \in \text { epi } H}\left(f(x)+g(y)-\left\langle x, x^{*}\right\rangle+\left\langle z, z^{*}\right\rangle\right) .
$$

Denoting the left term by $\alpha$ and the right one by $\beta$, and using again the fact that $g$ is increasing on $H(\operatorname{dom} H)+Q$, we have

$$
\begin{aligned}
\alpha & =\inf _{x \in \operatorname{dom} H} \inf _{y \in H(x)+Q}\left(f(x)+g(y)-\left\langle x, x^{*}\right\rangle\right) \\
& =\inf _{x \in \operatorname{dom} H}\left(f(x)+g(H(x))-\left\langle x, x^{*}\right\rangle\right)=-\varphi_{4}^{*}\left(x^{*}\right)
\end{aligned}
$$

and

$$
\beta=\inf _{x \in \operatorname{dom} H} \inf _{q \in Q, y \in Y}\left(f(x)+g(y)-\left\langle x, x^{*}\right\rangle+\left\langle H(x)+q-y, z^{*}\right\rangle\right) .
$$

It follows that $\beta=-\infty$ if $z^{*} \notin Q^{+}$. If $z^{*} \in Q^{+}$then

$$
\begin{aligned}
-\beta & =\sup _{x \in \operatorname{dom} H} \sup _{y \in Y}\left(\left\langle x, x^{*}\right\rangle-f(x)-\left(H(x), z^{*}\right\rangle+\left\langle y, z^{*}\right\rangle-g(y)\right) \\
& =\left(f+z^{*} \circ H\right)^{*}\left(x^{*}\right)+g^{*}\left(z^{*}\right) .
\end{aligned}
$$

As the inequality $\leq$ holds always in (18), it follows that (18) holds.

The inclusion $\supset$ holds always in (19) (see also [12, Proposition 4.4] for $\varepsilon=0$ ), while the converse inclusion follows from (18) by a standard argument. 
ReMARK 13. Combari, Laghdir and Thibault [13, Theorem 4.4, Proposition 5.4] obtain the conclusions of the preceding theorem (with $\varepsilon=0$ ) under conditions (i) and (vi) with ${ }^{i b} D$ replaced by ${ }^{i c} D$ and $g$ increasing. The case $f=0$ was considered by several authors: Kutateladze $[22,23]$ treats the algebraic case, Hiriart-Urruty [17, Theorem 5.1] obtains (19) under condition (iv) [for $Y=\mathbf{R}$ ], Zălinescu [46], [47, (6)], [51, Theorem 2.7.5] uses (iv) and, in [47, Theorem 4 (11)], [51, Theorem 2.7.5], a stronger form of (vi) [instead of epi $H$ is closed, $\operatorname{dom} H$ is closed and $\left.H\right|_{\operatorname{dom} H}$ is continuous (and $X_{0}=X$ in the first reference)], Lemaire [25, Proposition 2] obtains (19) for $\varepsilon=0$ using condition (iv). Note that the general case may be obtained from the one with $f=0$.

A final remark: the conditions using ${ }^{i c} D$ and $D^{i}$ (in Fréchet spaces) are, practically, equivalent; this is easy to see from the general approach by perturbation functions, but no so evident for the Fenchel duality. This fact was pointed in [50, Theorem 6], but we considered that it is not worth to detail all the situations treated in [46]. This was the case with the continuous versions of the problems treated in [46], too; we only sketched them in $\S 4$ of that paper (but the details may be found in the author's thesis, University of Iaşi, 1983).

\section{Acknowledgment}

The author thanks the anonymous referee for bringing to his attention the references $[11,20,24,26-28]$ and for the remarks which he tried to answer in the revised version. He also thanks Prof. D. Azé from University of Perpignan for sending a copy of [21]. Of course, all the results and comments related to these references are added in the revised version.

\section{References}

[1] H. Attouch and H. Brézis, "Duality for the sum of convex functions in general Banach spaces", in Aspects of Mathematics and its Applications (ed. J. Barroso), (Elsewier Science Publishers B.V., North Holland Amsterdam, 1986) 125-133.

[2] H. Attouch and M. Théra, "A general duality principle for the sum of two operators", J. Convex Anal. 3 (1996) 1-24.

[3] J. P. Aubin, Mathematical Methods of Game and Economic Theory (North-Holland, Amsterdam, 1979).

[4] D. Azé, "Duality for the sum of convex functionals in general normed spaces", Arch. Math. 62 (1994) 554-561.

[5] V. Barbu and T. Precupanu, Convexity and Optimization in Banach spaces (Editura Academiei Bucureşti and D. Reidel Publishing Company, Dordrecht, 1986). 
[6] J. F. Bonnans and R. Cominetti, "Perturbed optimization in Banach spaces I: A general theory based on a weak directional constraint qualification", SIAM J. Control Optimization 34 (1996) 1151-1171.

[7] J. M. Borwein, "Convex relations in analysis and optimization", in Generalized Concavity in Optimization and Economics (eds. S. Schaible and W. T. Ziemba), (Academic Press, New York, 1981) 335-377.

[8] J. M. Borwein, “Adjoint process duality", Math. Oper. Res. 8 (1983) 403-434.

[9] J. M. Borwein and S. Fitzpatrick, "Local boundedness of monotone operators under minimal hypotheses", Bull. Aust. Math. Soc. 39 (1988) 439-441.

[10] J. M. Borwein and A. S. Lewis, "Partially finite convex programming, Part I: Quasi relative interiors and duality theory", Math. Program. 57 (1992) 15-48.

[11] O. Chaldi, Z. Chbani and H. Riahi, "Directional derivatives and applications to subdifferential calculus", Preprint, University of Marrakech, 1997.

[12] C. Combari, M. Laghdir and L. Thibault, "Sous-différentiels de fonctions convexes composées", Ann. Sci. Math. Québec 18 (1994) 554-561.

[13] C. Combari, M. Laghdir and L. Thibault, "On subdifferential calculus for convex functions defined on locally convex spaces", to appear in Ann. Sci. Math. Québec.

[14] R. Cominetti, "Fenchel's theorem revisited", manuscript, 1988.

[15] I. Ekeland and R. Temam, Analyse Convexe et Problèmes Variationnels (Dunod, Gauthier-Villars, Parisi, 1974).

[16] M. S. Gowda and M. Teboulle, "A comparison of constraint qualifications in infinite-dimensional convex programming", SIAM J. Control Optimization 28 (1990) 925-935.

[17] J. B. Hiriart-Urruty, " $\varepsilon$-subdifferential calculus", in Convex Analysis and Optimization (eds. J. P. Aubin and R. B. Vinter) Research Notes in Mathematics 57 (Pitman, 1982) 43-92.

[18] V. Jeyakumar, "Duality and infinite dimensional optimization", Nonlinear Anal. TMA 15 (1990) 1111-1122.

[19] V. Jeyakumar and H. Wolkowicz, "Generalizations of Slater's constraint qualification for infinite convex programs", Math. Program. 57B (1992) 85-101.

[20] J.-L. Joly, "Une famille de topologies et de convergences sur l'ensemble des fonctionnelles convexes", Thes̀e d'ètat, Université de Grenoble, 1970.

[21] J.-L. Joly and P.-J. Laurent, "Stability and duality in convex minimization problems", R.I.R.O R-2 (1971) 3-42.

[22] S. S. Kutateladze, "Convex operators", Uspehi Mat. Nauk 34 (1979) 167-196, Russian.

[23] S. S. Kutateladze, “Convex $\varepsilon$-programming”, Dokl. Akad. Nauk SSSR 245 (1979) 1048-1050, Russian.

[24] P.-J. Laurent, Approximation et Optimisation (Hermann, Paris, 1972).

[25] B. Lemaire, "Subdifferential of a convex composite functional. Application to optimal control in variational inequalities", in Proceedings of IIASA Workshop on Nondifferentiable Optimization, Sopron 1984, Lecture Notes in Economics and Mathematical Systems, (Springer, Berlin, 1985).

[26] D. Mentagui, "Caractérisation de la stabilité d'un problème de minimisation associé à une fonction de perturbation particulière", Publ. Inst. Math., Nouv. 60 (74) (1996) 65-74.

[27] J.-J. Moreau, Fonctionnelles Convexes, Lecture Notes (Collège de France, Paris, 1966).

[28] M. Moussaoui and M. Volle, "Qausicontinuity and united functions in convex duality theory", Preprint 6, 1996, University of Avignon (to appear in Commun. Appl. Nonlinear Anal. 4 (1997)).

[29] J. P. Penot, “On the existence of Lagrange multipliers in nonlinear programming in Banach spaces", in Optimization and Optimal Control (eds. A. Auslender, W. Oettli and J. Stoer), Lecture Notes in Control and Information Sciences 30 (Springer-Verlag, Berlin, 1981) 89-104.

[30] R. R. Phelps, Convex functions, monotone operators and differentiability, Lecture Notes in Mathematics 1364 (Springer, Berlin, 1989). 
[31] J. Ponstein, Approaches to the Theory of Optimization (Cambridge Univ. Press, 1980).

[32] S. Robinson, "Regularity and stability for convex multivalued functions", Math. Oper. Res. 1 (1976) 130-143.

[33] R. T. Rockafellar, "Extensions of Fenchel's duality theorem for convex functions", Duke Math. J. 33 (1966) 81-90.

[34] R. T. Rockafellar, "Duality and stability in extremum problems involving convex functions", Pacific J. Math. 21 (1967) 167-187.

[35] R. T. Rockafellar, Convex Analysis (Princeton Univ. Press, Princeton, 1970).

[36] R. T. Rockafellar, Conjugate Duality and Optimization (SIAM Publications, Philadelphia, 1974).

[37] B. Rodrigues, "The Fenchel duality theorem in Fréchet spaces", Optimization 21 (1990) 13-22.

[38] B. Rodrigues and S. Simons, "Conjugate functions and subdifferentials in non-normed situations for operators with complete graphs", Nonlinear Anal. TMA 12 (1988) 1069-1078.

[39] S. Simons, "The occasional distributivity of $\circ$ over $\stackrel{+}{e}$ and the change of variable formula for conjugate functions", Nonlinear Anal., TMA 14 (1990) 1111-1120.

[40] S. Simons, "Sum theorems for monotone operators and convex functions", Trans. Am. Math. Soc. (to appear).

[41] M. Théra, "Subdifferential calculus for convex operators", J. Math. Anal. Appl. 80 (1981) 78-91.

[42] D. Tiba, "Subdifferential of composed functions and applications in optimal control", An. Sti. Univ. "Al. I. Cuza" Iaşi Sect. la Mat. 23 (1977) 381-386.

[43] M. Volle, "Some applications of the Attouch-Brezis condition to closedness criterions, optimization, and duality", (Seminaire d'Analyse Convexe, Montpellier, 1992), exposé n 16.

[44] C. Zălinescu, "A generalization of the Farkas lemma and applications to convex programming", $J$. Math. Anal. Appl. 66 (1978) 651-678.

[45] C. Zălinescu, "On an abstract control problem", Numer. Funct. Anal. Optimization 2 (1980) 531542.

[46] C. Zălinescu, "Duality for vectorial nonconvex optimization by convexification and applications", An. Sti. Univ. "Al. I. Cuza" Iaşi, Sect. la Mat 29 (3) (1983) 15-34.

[47] C. Zălinescu, "Duality for vectorial convex optimization, conjugate operators and subdifferentials. The continuous case", Abstract of a talk at the conference "Mathematical Programming - Theory and Applications", Eisenach 1984 (unpublished).

[48] C. Zălinescu, “On J. M. Borwein's paper 'Adjoint process duality”, Math. Oper. Res. 11 (1986) 692-698.

[49] C. Zălinescu, "Solvability results for sublinear functions and operators", Z. Oper. Res. 31 (1987) A79-A101.

[50] C. Zălinescu, “On some open problems in convex analysis”, Arch. Math. 59 (1992) 566-571.

[51] C. Zălinescu, Mathematical programming in infinite dimensional normed linear spaces (Editura Academiei, Bucharest, 1994) (in Romanian). 